\title{
Crashworthiness of Inflatable Thin-Walled Structures for Impact Absorption
}

\author{
Cezary Graczykowski and Jan Holnicki-Szulc \\ Institute of Fundamental Technological Research, Pawińskiego 5b, 02-106 Warsaw, Poland \\ Correspondence should be addressed to Cezary Graczykowski; cgraczyk@ippt.pan.pl
}

Received 15 June 2015; Revised 20 September 2015; Accepted 27 September 2015

Academic Editor: Roberto Montanini

Copyright (c) 2015 C. Graczykowski and J. Holnicki-Szulc. This is an open access article distributed under the Creative Commons Attribution License, which permits unrestricted use, distribution, and reproduction in any medium, provided the original work is properly cited.

\begin{abstract}
The paper describes application of innovative, inflatable thin-walled structures for absorption of the impact loading and thoroughly investigates their crash characteristics. The proposed concept assumes inflation of thin-walled structures with compressed gas of appropriately adjusted pressure in order to improve their basic mechanical properties, enhance energy dissipation capabilities, and increase corresponding durability to impact loading. In the first part of the paper the influence of compressed gas on mechanical characteristics of aluminium beverage can is analysed experimentally and by the corresponding numerical simulations. The following section proposes and numerically verifies three diverse engineering applications of inflatable thin-walled structures for impact absorption. Finally, the last part introduces the concept of adaptive inflatable barrier and briefly presents three simple strategies of pressure control. Both the performed basic experiment and the conducted numerical simulations show the advantageous influence of compressed gas and prove the feasibility of using inflatable thin-walled structures for impact absorption.
\end{abstract}

\section{Introduction}

Thin-walled structures are commonly used in transport and mechanical industry because of their large stiffness, durability, and small weight. Additionally, thin-walled structures made of aluminium or steel effectively absorb the energy of axial loading due to the process of plastic folding. The above features cause that thin-walled elements are efficiently applied in crushing zones of trains, cars, and other energy absorbing structures.

Mechanics of thin-walled structures subjected to large deformations can be analysed by both analytical and numerical methods. Despite many complex phenomena which occur during crushing of thin-walled structures (including material and geometrical nonlinearities such as plasticity, buckling, and contact) many analytical and semiempirical solutions were derived thanks to application of simplifying assumptions concerning material models and kinematics of deformation; compare well-known solution for axisymmetric crushing of circular tube by Alexander [1]. Comprehensive approach to mechanics of thin-walled profiles, methods of determination of their folding patterns, and estimation of impact absorption capabilities were presented in books by Jones [2] and conference proceedings by Jones and Wierzbicki [3-5]. The research in the field of thin-walled structures had also influenced development and stimulated progress in design of complex crashworthy structures such as passenger vehicles, trains, and ships [6].

The numerical approach to impact problems was possible owing to contemporary understanding of structural dynamics and development of finite element method as an efficient tool for solving complex problems of nonlinear mechanics $[7,8]$. The recent finite element codes dedicated to crashworthiness problems [9] utilise explicit methods for integrating equations of motion [10,11]. The choice of explicit methods is motivated by the ease of their numerical implementation and the possibility of obtaining efficient and robust solution of strongly nonlinear crashworthiness problems. On the other hand, the main drawback of explicit methods is the requirement of using relatively short time steps in order to ensure numerical stability and the existence of critical time step beyond which numerical instabilities occur. Thus, in case 
of rigorous stability conditions leading to extremely short time step size the explicit methods are sometimes replaced by the implicit ones. The implicit methods have, however, their own limitations including more difficult implementation for strongly nonlinear problems, complex computations at a single time step, and common difficulties with convergence of the numerical solution.

In addition to the above classical numerical methods the alternative so-called semianalytical approaches to crashworthiness problems were proposed and developed. The methods of this group provide substantial simplification of the crashworthiness problem and corresponding reduction of its computational cost. The examples are the methods based on discretisation of the structure into the so-called macroelements with predefined folding patterns developed by Abramowicz [13].

Despite many years of research and development the mechanical properties and energy absorption capabilities of thin-walled absorbers of various shapes and cross sections as well as their practical applications are still studied, for example, by Kim and Wierzbicki $[14,15]$ or Han and Yamazaki [16]. A full review of various types of conventional thinwalled impact energy absorbers will not be performed here, since it can be found in subject literature, for example, in comprehensive paper by Alghamdi [17], Ph.D. thesis by Lee [18], or Zhang [19]. Instead, the attention will be focused on innovative methods of enhancement and control of crashworthy capabilities of thin-walled structures.

Crashworthiness of thin-walled structures subjected to axial loading was improved in various manners. In particular, filling thin-walled structure with granular material (like sand or grain) and taking the advantage of friction forces generated between granules during impact were proposed by Lee [18]. In turn, Zhang [19] examined the usage of buckling initiators activated just before expected impact in order to reduce initial peak of crushing force. Moreover, thin-walled absorbers composed of two sections joined by pyrotechnic connectors, which can be detached during impact in order to reduce global stiffness, were proposed and tested experimentally by Ostrowski et al. [20]. Another important concept is filling axially loaded circular tubes with compressed gas in order to take advantage of the effect of gas compression during impact and to affect the shape of deformation of thin-walled absorber; compare Zhang [19]. The above concept was also studied by Greń [21] who had developed precise analytical model of the process of longitudinal crushing assisted by gas pressure. Finally, the application of the above concept to control stiffness of the vehicle longitudinal frontal members was studied by Pipkorn and Håland [22].

In contrast to previously mentioned solutions, the concept presented in this paper is focused on thin-walled structures subjected to lateral impact. In case of lateral loading, thin-walled structure easily undergoes buckling and local plastic yielding and, as a result, only a small part of the impact energy is dissipated. However, as it will be shown in the following sections, filling thin-walled structure with compressed gas of properly adjusted pressure may significantly improve its mechanical properties and increase global durability to impact load. The paper starts with a simple experiment where aluminium beverage can is subjected to the action of transverse force and the influence of internal pressure on buckling phenomenon is observed. In addition, mechanical response of the inflated can is studied with the use of various numerical models. Further, three diverse applications of inflatable thin-walled structures for impact absorption are proposed. The corresponding simulations of impact process are conducted and general conclusions concerning the effects of structure inflation are drawn. Finally, the last section proposes the concept of adaptive inflatable barrier with real-time pressure control. The corresponding simplified two-dimensional model is applied to demonstrate the potential of compressed gas in minimizing generated internal forces, reducing impacting object deceleration, and obtaining desired final deformation of the structure.

Several ideas presented within this paper are included in the patent claim [23], while initial and immature versions of the proposed concepts were presented in conference paper [24] dedicated to buckling of the aluminium can and design of the inflatable barrier.

\section{Basic Experiment: Buckling of Inflated Thin-Walled Can}

Basic experiment confirming the beneficial influence of filling thin-walled structure with compressed gas was performed with the use of aluminium beverage can (the experiment was performed by Mr. Rafał Chmielewski, who is gratefully acknowledged for his work). In the conducted tests the right end of the empty can was clamped around the circumference, while the left end was reinforced by special ring and subjected to action of vertical force $\mathbf{F}$ acting upwards (Figure 1). As a result of applied boundary conditions and external force, the can was acting as a cantilever. The applied loading caused bending of the can, tension of its lower wall, and compression of the upper one.

In the initial experiment the can was not sealed so internal pressure was permanently equal to ambient pressure. During the performed test the value of vertical force was gradually increased. Sudden collapse of the structure was observed when vertical force reached $155 \mathrm{~N}$ and it was caused by buckling of cylinder sidewalls. The buckling region covered a large part of the can located between its middle part and the support (Figure 1(a)). Moreover, buckling shape was approximately symmetrical on both sides of the cylinder. The collapse of the structure occurred at relatively small displacement of the left edge, which indicates small work done by external force before buckling.

In the second stage of the experiment the can was sealed and inflated with compressed gas to pressures of $0,2 \mathrm{MPa}$, $0,4 \mathrm{MPa}, 0,6 \mathrm{MPa}$, and 0,8 MPa. During the experiment the pressure of gas was not externally changed or controlled. The applied internal pressure was expected to cause increase of the critical force since it generates additional surface loading, which acts against buckling phenomenon. Two particular effects of internal pressure, which can be distinguished, are as follows: 


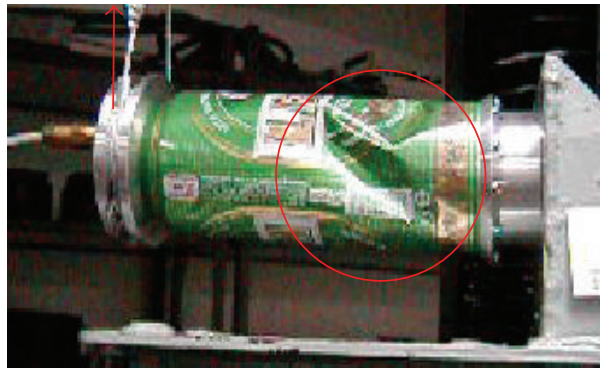

(a)

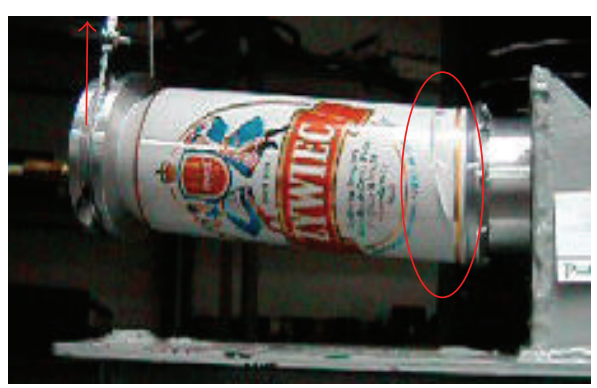

(b)

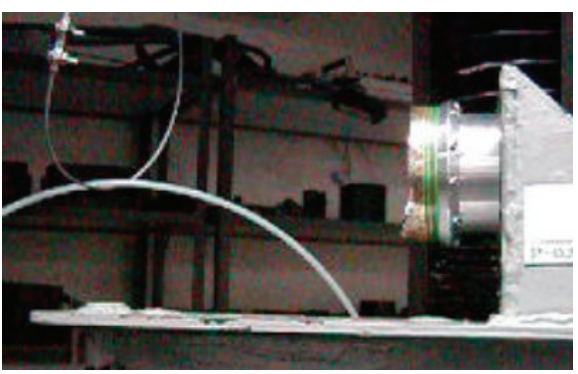

(c)

Figure 1: Deformation of the beverage can with overpressure: (a) $0 \mathrm{MPa}$, (b) 0,6 MPa, and (c) 0,8 MPa.

(i) reduction of compressive stresses arising at prone-tobuckling upper wall,

(ii) counteracting inward deformation which occurs during buckling of the empty can.

According to the above expectations, the value of critical force causing buckling of the structure was gradually rising (up to $610 \mathrm{~N}$ for internal pressure of $0,6 \mathrm{MPa}$ ). Along with an increase of critical force, the area of buckling was decreasing and moving into the direction of the support, Figure 1(b). Moreover, the buckling phenomenon had occurred at apparently larger displacement of the left end of the can which indicates significant increase of work done by external force and corresponding increase of energy absorbed by the inflated can.

In the last considered case of pressure of $0,8 \mathrm{MPa}$, the maximal value of applied force was approximately equal to $700 \mathrm{~N}$; however a sudden burst of the can had occurred briefly after buckling because of material rapture in the vicinity of the support (Figure 1(c)). Unfortunately the limitations of the conducted experiment, in particular insufficient quality and frame rate of applied camera, did not allow identifying the location where material rapture and corresponding burst were initiated. Thus, the only available data concerning the form of can destruction was circumferential shape of can rupture in the vicinity of the support (Figure 1(c)) and irregular rapture of disconnected part in longitudinal direction (not presented).

The explanation of the phenomenon of sudden burst of the can at the end of the process may be twofold. The most direct hypothesis is that burst was initiated in the region located at the bottom of the can in the vicinity of the support and it was caused by exceeding maximal allowable value of tensile stress, which induced peripherally propagating rupture of the material. According to alternative concept the burst was initiated at a certain point of the buckling area, for example, top or side of the can where buckling phenomenon is manifested by distinct indentations (cf. Figure 1(b)), which can provoke the occurrence of material rupture.

The experiment reveals two important features of the inflatable thin-walled structures. Primarily, the durability of thin-walled structures to lateral loading and the amount of dissipated energy can be substantially increased by the use of compressed gas. On the other hand, application of excessive internal pressure is related to a danger of sudden destruction or blast of the structure. This implicates the necessity of precise adjustment of the initial value of pressure or even the requirement of its real-time control during the process of deformation.

\section{Numerical Analysis of Inflated Thin-Walled Can}

The problem of bending of inflated can was simulated numerically with the use of finite element method. The interaction of the internal gas and the structure was modelled by applying uniformly distributed surface loading perpendicular to the surface of the can. Since in considered simulations the internal volume of the can (and thus the volume of gas) changes insignificantly the corresponding changes of gas pressure are expected to be negligible. Consequently, the action of internal pressure was modelled by distributed loading of a constant value. The following numerical analyses were conducted:

(1) static analysis of bending of empty and inflated can, 
(2) linear buckling analysis of empty and inflated can during bending,

(3) nonlinear analysis of bending, buckling, and bursting of the can,

(4) linear buckling analysis of the can during axial compression,

(5) modal analysis of empty and inflated can.

Thin-walled cylindrical shell structure considered in numerical simulations had the dimensions of the aluminium can: length $l=170 \mathrm{~mm}$, radius of the base $r=33 \mathrm{~mm}$, and thickness of the wall $t=0,1 \mathrm{~mm}$. Thickness of both bases of the cylinder was equal to $0,5 \mathrm{~mm}$ to model large stiffness of the reinforcing ring from the experiment. Since the imperfections of the can from cylindrical shape and indentations of its lateral walls, which substantially decrease global bending resistance of the structure, are probabilistic in nature and hard for estimation they were not directly introduced into the model. Instead, their influence on the response of the can was modelled in a simplified way by decreasing the standard value of aluminium Young modulus by $20 \%$ (from $70 \mathrm{GPa}$ to $56 \mathrm{GPa}$ ). Such approach provides agreement of the numerical analysis and the buckling experiment for the cases of both empty and inflated aluminium can.

The main solver applied for the numerical simulations was commercial finite element code ABAQUS (both Standard and Explicit) and the main finite element applied in simulations was doubly curved thin shell element with reduced integration, hourglass control, and finite membrane strains (S4R). Different sizes of finite elements were used depending on the type of conducted analysis.

3.1. Static Analysis. Static analysis of bending of the inflated can was aimed at investigating the influence of internal pressure on distribution of internal stresses in cylinder walls. Internal pressure was modelled as distributed loading applied perpendicularly to all internal walls of the cylinder. In turn, the applied vertical load was distributed along the circumference of the left base of the cylinder. At this stage of analysis, characteristics of the material were assumed as linear elastic ones; however, the equilibrium equations were considered in actual configuration.

The problem of bending of the inflated can consists of the step of inflation (the increase of pressure loading) and the step of bending (the increase of external force). In finite element notation the problem solved reads as follows:

$$
\begin{aligned}
& \text { Step 1: } \mathbf{K}\left(\mathbf{Q}_{\mathbf{p}}, \mathbf{q}\right) \mathbf{q}=\mathbf{Q}_{\mathbf{p}}(p, \mathbf{q}) \text {, } \\
& \text { Step 2: } \mathbf{K}\left(\mathbf{Q}_{\mathbf{p}}^{\max }, \mathbf{Q}_{\mathbf{F}}, \mathbf{q}\right) \mathbf{q} \\
& \quad=\mathbf{Q}_{\mathbf{p}}\left(p^{\max }, \mathbf{q}\right)+\mathbf{Q}_{\mathbf{F}}(F, \mathbf{q}),
\end{aligned}
$$

where $\mathbf{K}$ is the stiffness matrix dependent on actual applied loading $\mathbf{Q}$ and actual deformation of the structure $\mathbf{q}$. The quantity $\mathbf{Q}_{\mathbf{p}}(p, \mathbf{q})$ is the load vector caused by internal pressure $p$ (in particular $\mathbf{Q}_{\mathbf{p}}^{\max }=\mathbf{Q}_{\mathbf{p}}\left(p^{\max }, \mathbf{q}\right)$ ) and $\mathbf{Q}_{\mathbf{F}}(F, \mathbf{q})$ is the load vector caused by external force $\mathbf{F}$ of a magnitude $F$. Both load vectors depend on actual deformation of the structure since pressure loading is perpendicular to the walls of the can and external force is assumed to follow the structure deformation. Let us note that the case when both forces are applied simultaneously

$$
\mathbf{K}\left(\mathbf{Q}_{\mathbf{p}}, \mathbf{Q}_{\mathbf{F}}, \mathbf{q}\right) \mathbf{q}=\mathbf{Q}_{\mathbf{p}}(p, \mathbf{q})+\mathbf{Q}_{\mathbf{F}}(F, \mathbf{q})
$$

is not equivalent to problem defined by (1a), (1b), which is reflected in different arguments of the stiffness matrix and consequently different path of structure equilibrium. Significant simplification of the above problem is obtained by setting equilibrium equations in initial (nondeformed) configuration. In such a case the problem solved reads

$$
\mathbf{K q}=\mathbf{Q}_{\mathbf{p}}(p)+\mathbf{Q}_{\mathbf{F}}(F)
$$

and can be decomposed into two simpler problems:

$$
\begin{aligned}
\mathbf{K q}_{\mathbf{p}} & =\mathbf{Q}_{\mathbf{p}}(p), \\
\mathbf{K q}_{\mathbf{F}} & =\mathbf{Q}_{\mathbf{F}}(F), \\
\mathbf{q} & =\mathbf{q}_{\mathbf{p}}+\mathbf{q}_{\mathbf{F}},
\end{aligned}
$$

where $\mathbf{q}_{\mathbf{p}}$ and $\mathbf{q}_{\mathbf{F}}$ indicate displacements caused by internal pressure and vertical force. The total displacement $\mathbf{q}$ can be calculated as a sum of the two above displacements according to the superposition principle. Equations (4a), (4b) reveal, in a simplified manner, the influence of internal pressure on distribution of internal forces in considered structure and corresponding shape of deformation.

The corresponding numerical simulations were conducted with the use of ABAQUS Standard. The exemplary numerical results are related to the case of bending force equal to $165 \mathrm{~N}$ (the value of buckling force obtained from numerical linear buckling analysis, cf. Section 3.2) and two values of internal overpressure: $0 \mathrm{MPa}$ and $0,4 \mathrm{MPa}$. In case of zero internal overpressure, the distribution of longitudinal stress is very regular with tension region at the lower side and compression regions at the upper side of the cylinder (Figure 2(a)). Although deformation of the cylinder is relatively small, the influence of geometry change on distribution of internal forces is reflected in slightly unsymmetrical distribution of longitudinal stress on the bottom and on the top of the cylinder.

In turn, the loading caused by application of internal pressure results in nearly uniform longitudinal and circumferential tensile stresses in the sidewalls of the cylinder (except the peripheral regions of clamping and free end). Consequently, the interaction of internal pressure and bending force leads to reduction of longitudinal compressive stresses in the upper part of the cylinder with simultaneous increase of longitudinal tensile stresses in the lower part (Figure 2(b)).

Although the finite element analysis reveals vague effect of bending of cylinder walls (slight variation of stress across shell thickness), the distribution of stresses can be estimated by means of membrane shell theory [25], which assumes that equilibrium of shell is provided without the presence of bending forces. Analytical formulae defining stress in 


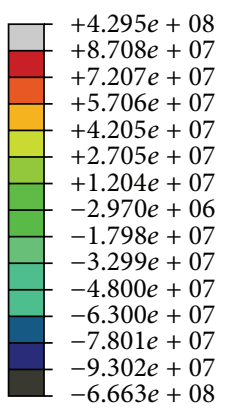

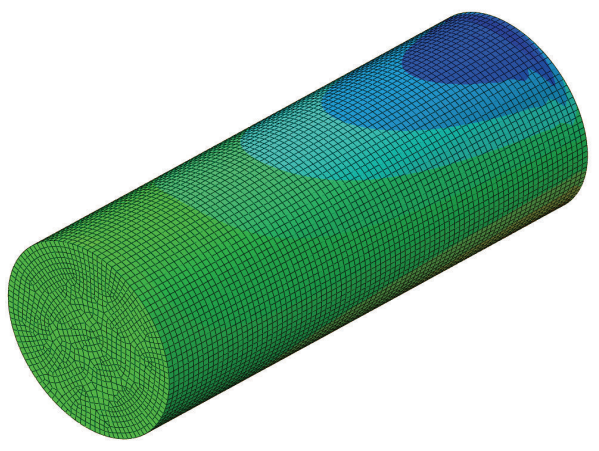

(a)
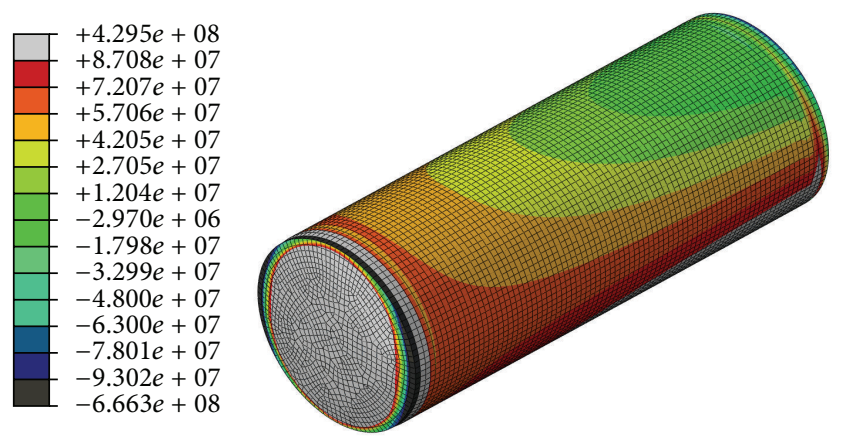

(b)

FIGURE 2: Distribution of the longitudinal stress [Pa] at outer side of the shell caused by bending force of $165 \mathrm{~N}$ : (a) empty can, (b) internal overpressure of 0,4 .

the longitudinal direction at critical points on the top and bottom of the cylinder in the vicinity of the support read as follows.

(1) First Load Case. The case with force acting at the end of the cantilever $F=165 \mathrm{~N}$ and no internal overpressure is

$$
\begin{aligned}
\sigma_{\text {top }} & =-\frac{M r}{J}=-\frac{F l r}{(\pi / 4)\left(r^{4}-(r-t)^{4}\right)} \\
& =-82,36 \mathrm{MPa}, \\
\sigma_{\text {bottom }} & =82,36 \mathrm{MPa} .
\end{aligned}
$$

(2) Second Load Case. The case with force $F=165 \mathrm{~N}$ and internal overpressure $p=0,4 \mathrm{MPa}$ is

$$
\begin{aligned}
\sigma_{\text {top }} & =-\frac{M r}{J}+\frac{p r}{2 t}=-16,4 \mathrm{MPa}, \\
\sigma_{\text {bottom }} & =\frac{M r}{J}+\frac{p r}{2 t}=148,5 \mathrm{MPa} .
\end{aligned}
$$

In the above formulae $M$ is the bending moment caused by applied force, while $J$ is the moment of inertia of the circular cross section, which can be expressed in terms of radius of the can $r$ and thickness of the wall $t$. Both the above results are in good agreement with the results of FEM simulations presented in Figure 2. The obtained results indicate that observed in the experiment buckling of the empty can occurs at relatively low value of stress (below plastic limit of the aluminium) and the process can be treated as elastic buckling. On the other hand, when the cylinder is subjected to action of internal pressure of $0,8 \mathrm{MPa}$ and bended by the force of $700 \mathrm{~N}$ (extreme conditions in the experiment) the maximal longitudinal stress calculated according to (6) equals $481 \mathrm{MPa}$ and exceeds yield strength of aluminium. Therefore, the phenomenon obtained in the experiment in case of high internal pressure should be rather treated as elastoplastic buckling.

3.2. Linear Buckling Analysis. The next stage of considerations was linear buckling analysis of empty and inflated can during bending by the vertical force. Analysis of buckling of inflated structure requires two subsequent steps:

(i) initial prestressing where distributed loading modelling gas pressure is applied,

(ii) eigenvalue buckling analysis where critical value of the bending load is searched.

The equations governing two parts of the problem read as follows:

$$
\begin{gathered}
\text { Step 1: } \mathbf{K} \mathbf{q}_{\mathbf{p}}=\mathbf{Q}_{\mathbf{p}} \\
\text { or } \mathbf{K}\left(\mathbf{Q}_{\mathbf{p}}, \mathbf{q}_{\mathbf{p}}\right) \mathbf{q}_{\mathbf{p}}=\mathbf{Q}_{\mathbf{p}}, \\
\text { Step 2: }\left[\mathbf{K}\left(\mathbf{Q}_{\mathbf{p}}^{\max }\right)-\lambda \mathbf{K}_{\mathbf{Q}_{\mathbf{F}}}\right] \mathbf{v}=\mathbf{0} \\
\text { or }\left[\mathbf{K}\left(\mathbf{Q}_{\mathbf{p}}^{\max }, \mathbf{q}_{\mathbf{p}}\right)-\lambda \mathbf{K}_{\mathbf{Q}_{\mathrm{F}}}\left(\mathbf{q}_{\mathbf{p}}\right)\right] \mathbf{v}=\mathbf{0} .
\end{gathered}
$$

The first step of simulation is a standard static analysis, which can be executed either as a geometrically linear or as a nonlinear one. The result of this analysis is prestressed state corresponding to initial or deformed configuration of the structure. The second step of simulation is linear buckling analysis performed either for prestressed nondeformed configuration defined by stiffness matrix $\mathbf{K}\left(\mathbf{Q}_{\mathbf{p}}^{\max }\right)$ or, alternatively, for deformed configuration defined by stiffness matrix $\mathbf{K}\left(\mathbf{Q}_{\mathbf{p}}^{\max }, \mathbf{q}_{\mathbf{p}}\right)$. In buckling analysis the global stiffness matrix is composed of base stiffness matrix $\mathbf{K}$ and load stiffness matrix $\mathbf{K}_{\mathbf{Q}_{\mathrm{F}}}$ corresponding to external vertical load of a unit value. The step is aimed at finding value of external bending load for which (7b) has nontrivial solutions, that is, for which global stiffness matrix of the system becomes singular. The eigenvalues $\lambda$ are determined as solution of the equation:

$$
\begin{array}{r}
\operatorname{det}\left[\mathbf{K}\left(\mathbf{Q}_{\mathbf{p}}^{\max }\right)-\lambda \mathbf{K}_{\mathbf{Q}_{\mathbf{F}}}\right]=\mathbf{0} \\
\text { or } \operatorname{det}\left[\mathbf{K}\left(\mathbf{Q}_{\mathbf{p}}^{\max }, \mathbf{q}_{\mathbf{p}}\right)-\lambda \mathbf{K}_{\mathbf{Q}_{\mathbf{F}}}\left(\mathbf{q}_{\mathbf{p}}\right)\right]=\mathbf{0}
\end{array}
$$

which further allows finding value of critical bending loading $\lambda \mathbf{Q}_{\mathbf{F}}$, total value of critical loading $\mathbf{Q}_{\mathbf{p}}+\lambda \mathbf{Q}_{\mathbf{F}}$, and eigenvectors $\mathbf{v}$. 

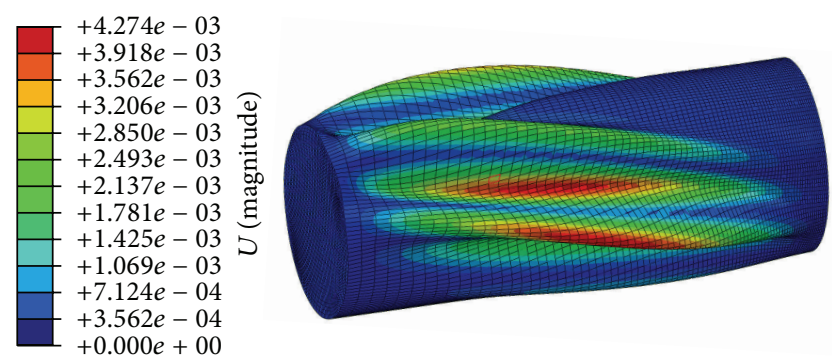

(a)

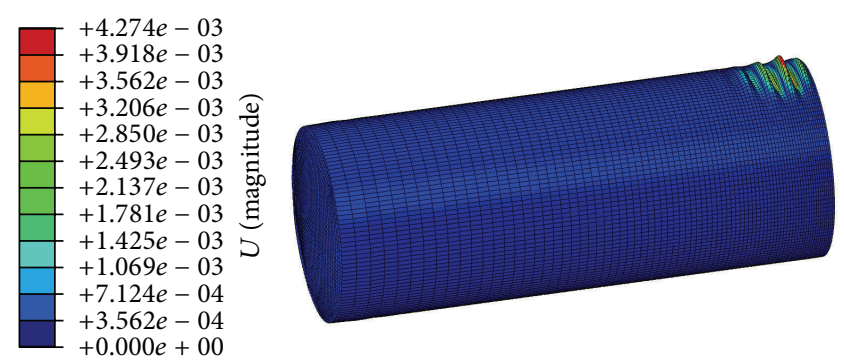

(b)

FIgURE 3: Change of buckling shape as a result of increase of internal overpressure in the cylinder: (a) $p=0 \mathrm{MPa},(\mathrm{b}) p=0,35 \mathrm{MPa}$.

The corresponding numerical simulations were conducted with the use of two finite element solvers (ABAQUS Standard and ANSYS Mechanical) where four- and eightnode shell elements were applied. In case of noninflated cylinder the value of the critical bending force corresponding to the first buckling mode computed by the two above solvers was equal to $171 \mathrm{~N}$ and $165 \mathrm{~N}$, which is only slightly higher than the value of critical force obtained from the experiment. Determined buckling shape (Figure 3(a)) includes longitudinal deformations arising on both sides of the cylinder. The fact that the subsequent values of the critical forces are close to each other indicates that the loss of stability of real structure may involve combination of several initial buckling shapes from the numerical analysis. The buckling shape obtained from the numerical simulations is slightly different than the deformation shape obtained during the experiment; however in both cases the deformation occurs along the longitudinal direction of the cylinder.

In the next step, the simulation of buckling was performed for several values of internal pressure inside the cylinder. The increase of pressure in subsequent numerical analyses had caused change of buckling shape (Figures 3(a) and 3(b)) and corresponding increase of critical vertical force (Figure 4). For the pressure range of $0-0,05 \mathrm{MPa}$ the first buckling shape covers a major part of the cylinder sidewalls. However, in the case when internal pressure is higher, the shape of buckling significantly changes. The area of buckling is significantly decreased and it is located exclusively in the vicinity of the support. In case of inflated structure a buckling phenomenon is associated with a smaller overall deformation of the structure and thus buckling itself can be considered as less dangerous for the structure operation.

The increase of internal pressure is associated with the raise of critical bending force causing can buckling. The computed value of critical force increases fast in a low range of internal pressure but further its growth is diminished and remains approximately linear; see Figure 4 . On the other hand, the increase of internal pressure is associated with decrease of the value of bending force which causes exceeding of tensile strength of the material at bottom wall $(500 \mathrm{MPa})$ and initiation of bursting. This effect was investigated by using linear static analysis, exactly the analytical model based on (6), which allowed calculating the corresponding critical value of force in terms of increasing pressure; see Figure 4.

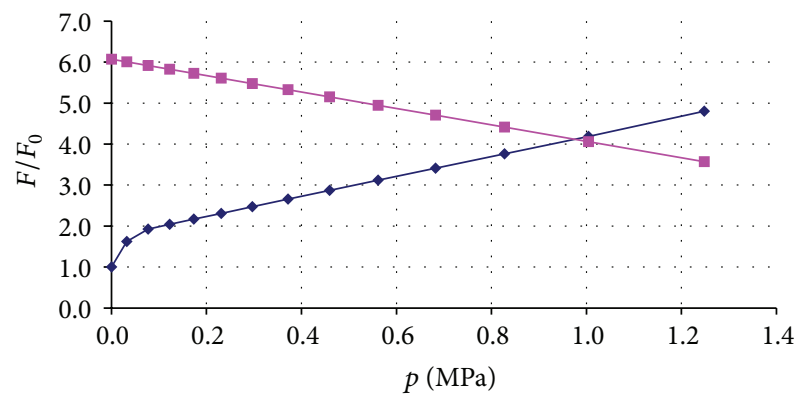

FIGURE 4: Force causing buckling of the can (increasing function) and force causing bursting of the can (decreasing function) in terms of gas pressure (both divided by initial buckling force $F_{0}=165 \mathrm{~N}$ ).

Results of the analysis performed for pressures $0-1,3 \mathrm{MPa}$ reveal that two ranges of pressure can be distinguished. When initial pressure is in the range $0-0,975 \mathrm{MPa}$ buckling of the can occurs at lower value of vertical force than bursting of the bottom wall. The point of intersection of both curves indicates theoretical optimal pressure of $0,975 \mathrm{MPa}$ at which buckling of the top wall and bursting of the bottom wall of the cylinder occur theoretically simultaneously. The vertical force that can be applied to the structure equals $678 \mathrm{~N}$ and it is 4,1 times larger than force causing collapse of noninflated structure $\left(F_{0}=165 \mathrm{~N}\right)$. Finally, when internal pressure is higher than optimal value of $0,975 \mathrm{MPa}$ the buckling of the cylinder does not occur and its destruction is caused by bursting of the bottom wall.

3.3. Nonlinear Analysis of Bending and Buckling. Nonlinear analysis of bending and buckling of the can was performed in order to verify results from linear buckling analysis and to analyse the postbuckling behaviour of the can with large deformation and nonlinear characteristics of the material. In case of nonlinear analysis, no special procedure for capturing the buckling phenomenon is required. Nevertheless, the buckling force at which sudden increase of deformation occurs can be clearly observed.

The analysis of can bending was conducted as a dynamic analysis involving inertial and damping forces. Similarly as in case of static analysis the numerical procedure was composed 
TABLE 1: Comparison of buckling forces obtained from linear buckling analysis (ABAQUS Standard) and nonlinear buckling analysis (ABAQUS Explicit).

\begin{tabular}{lcccccc}
\hline & $0 \mathrm{MPa}$ & $0,1 \mathrm{MPa}$ & $0,3 \mathrm{MPa}$ & $0,5 \mathrm{MPa}$ & $0,7 \mathrm{MPa}$ & $0,9 \mathrm{MPa}$ \\
\hline ABAQUS STD & $171,2 \mathrm{~N}$ & $270,4 \mathrm{~N}$ & $340,3 \mathrm{~N}$ & $410,5 \mathrm{~N}$ & $480,8 \mathrm{~N}$ & $551,2 \mathrm{~N}$ \\
ABAQUS XPL & $\sim 191 \mathrm{~N}$ & $\sim 280 \mathrm{~N}$ & $\sim 404 \mathrm{~N}$ & $\sim 506 \mathrm{~N}$ & $\sim 645 \mathrm{~N}$ & $\sim 779 \mathrm{~N}$ \\
\hline
\end{tabular}

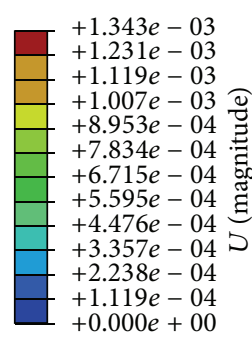

$+0.000 e+00$

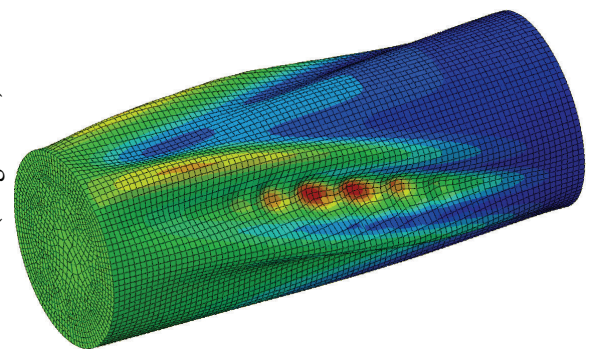

(a)

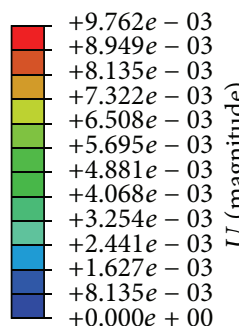

$+8.135 e-03$

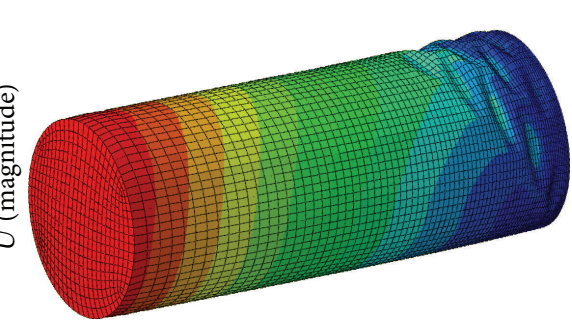

(b)

FIGURE 5: Buckling shapes obtained from nonlinear explicit dynamic analysis: (a) $p=0 \mathrm{MPa}$, (b) $p=0,3 \mathrm{MPa}$.

of initial step when the can is prestressed by internal pressure and the main step when it is bended by vertical force:

$$
\begin{aligned}
& \text { Step 1: } \mathbf{M} \ddot{\mathbf{q}}+\mathbf{C} \dot{\mathbf{q}}+\mathbf{K}\left(\mathbf{Q}_{\mathbf{p}}, \mathbf{q}\right) \mathbf{q}=\mathbf{Q}_{\mathbf{p}}(p(t), \mathbf{q}), \\
& \qquad t \in\left\langle 0, t_{1}\right\rangle, \\
& \text { Step 2: } \mathbf{M} \ddot{\mathbf{q}}+\mathbf{C} \dot{\mathbf{q}}+\mathbf{K}\left(\mathbf{Q}_{\mathbf{p}}^{\max }, \mathbf{Q}_{\mathbf{F}}, \mathbf{q}\right) \mathbf{q} \\
& \quad=\mathbf{Q}_{\mathbf{p}}\left(p\left(t_{1}\right), \mathbf{q}\right)+\mathbf{Q}_{\mathbf{F}}(F(t), \mathbf{q}), \quad t \in\left\langle t_{1}, t_{2}\right\rangle .
\end{aligned}
$$

The corresponding numerical simulations were performed by using dynamic explicit solver (ABAQUS Explicit) in order to avoid difficulties related to convergence of the analysis conducted by implicit method. The time scale was selected as sufficiently long in order to neglect the inertia of the structure but simultaneously not excessively long in order to provide reasonable computation time. In the initial analysis, the characteristics of material were assumed as linear elastic ones in order to obtain correspondence with preceding linear buckling analysis. Because of relatively long analysis time and resulting small influence of inertial and damping forces the governing equations are practically identical to (1a), (1b) corresponding to the static case.

The results obtained by means of the presented methodology confirm, in general, the previous results from the linear buckling analysis. In the case when the can is not inflated or when the value of pressure is low the buckling occurs at a large area of cylinder sidewalls, symmetrically on both sides, Figure 5(a). The computed value of buckling force for an empty cylinder was $11,9 \%$ higher than the value obtained from linear buckling analysis conducted with the same model in the same commercial FEM software; see Table 1. Moreover, it was observed that buckling phenomenon starts when vertical displacement of loaded edge of the cylinder equals approximately $0,8 \mathrm{~mm}$. The fact that prebuckling displacement is relatively small, but not negligible, indicates that linear buckling analysis constitutes acceptable

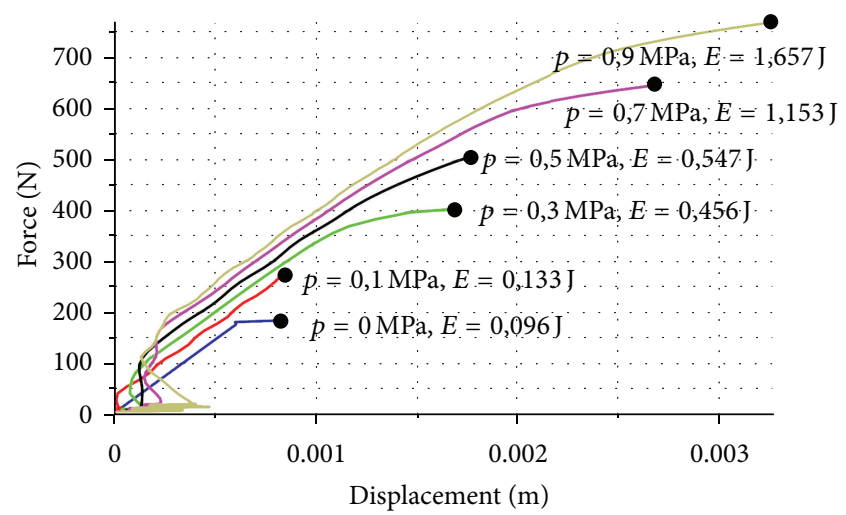

Figure 6: Applied force versus displacement of the free end of the can for various initial pressures.

approximation of the buckling phenomenon, but on the other hand it explains certain difference in values of buckling forces computed by the two proposed methods.

For pressures of $8-9 \mathrm{kPa}$ and higher the shape of buckling changes and concentrates at the vicinity of the support, Figure 5(b). In all these cases, the buckling force of the inflated cylinder obtained from the explicit dynamic analysis is higher than the value from linear buckling analysis, Table 1. Moreover, the increase of internal pressure causes that the displacement at which buckling occurs gradually rises and it reaches 3,5 $\mathrm{mm}$ for pressure equal to $0,9 \mathrm{MPa}$. The increase of prebuckling displacement justifies the increase of difference between the values of critical force obtained by linear and nonlinear bucking analysis. For the largest considered values of pressure, the linear buckling analysis can be used only for rough approximation of the real buckling force.

For each value of internal pressure the dependence between applied external loading and magnitude of displacement of the free end of the can was investigated. In Figure 6 each force-displacement curve is plotted until the 


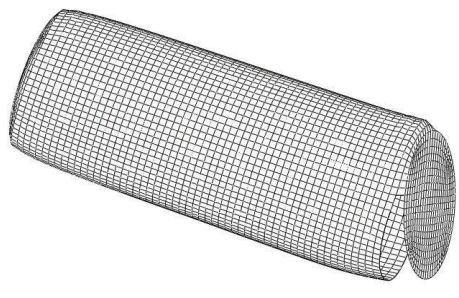

(a)

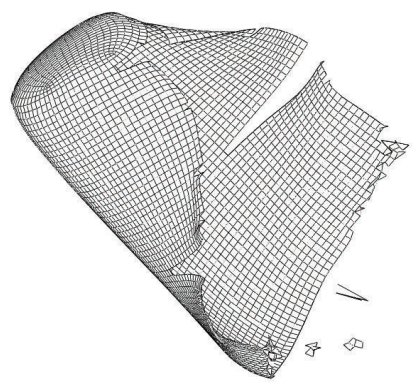

(b)

Figure 7: Two stages of simulation of bursting of the can: (a) initiation of bursting $F=711 \mathrm{~N}$, (b) total destruction of the can.

point where buckling of the can is initiated. As it can be clearly observed the inflation of the can causes increase of the maximal displacement and increase of the corresponding maximal value of force. The area below each curve denotes work done by applied loading, which equals the raise of strain energy of the structure. The conducted analysis indicates that the amount of energy which can be transferred to the structure before the buckling point increases from $0,096 \mathrm{~J}$ for an empty can up to 1,657 J for a can inflated to $0,9 \mathrm{MPa}$ (more than seventeenfold difference).

The last step of numerical investigation was an attempt of simulation of bursting of the can inflated with high internal pressure $(0,8 \mathrm{MPa})$, which strongly promotes tensile failure of the bottom wall of the can during bending by vertical force. Due to the lack of exact material for the aluminium utilised for production of thin-walled aluminium beverage cans (in particular its yields stress level and ultimate strength) the material data was adjusted in order to obtain the satisfactory qualitative and quantitative correspondence with the results of the conducted experiment (cf. Section 2). The elastic stage of deformation is assumed to occur in the range $0-200 \mathrm{MPa}$ ( $E=56 \mathrm{GPa})$, while plastic stage of deformation is assumed to occur in the range $200-400 \mathrm{MPa}$ (with maximal strain equal to 0,4$)$. Moreover, the rupture of the material is assumed to occur at $300 \mathrm{MPa}$.

The results of the numerical simulation (Figure 7) correspond to the first hypothesis concerning initiation of the burst, which assumes exceeding of the maximal tensile stress at the bottom of the can (cf. Section 2). In the numerical simulation the rupture of material starts at the lower side of the can in the vicinity of support and it propagates symmetrically towards its top until complete disconnection from the clamped end. During the process of burst the disconnected part of the can is irregularly torn in the longitudinal direction. Eventually, the final form of cylinder destruction obtained from the numerical simulation clearly resembles the results of the conducted experiment. In addition, the critical force at which bursting initiates is equal to $711 \mathrm{~N}$, which is very close to the value obtained from the experiment.

3.4. Linear Analysis of Buckling during Axial Compression. Apart from the above numerical simulations related to the conducted experiment two additional simulations were performed:

(i) linear analysis of buckling of empty and inflated can during axial compression,

(ii) modal analysis of empty and inflated can.

The problem of buckling of cylindrical shells under axial loading is widely considered in classical literature concerning shell structures, for example, in the book by Flugge [26]. The phenomenon of buckling of free-supported cylindrical shell under axial loading and pressure can be described fully analytically by assuming sinusoidal buckling shapes and introducing them into differential equations of shell buckling. Finally, the formula for determination of the nondimensional parameter $q_{2}$ defining axial loading in terms of number of half-waves along cylinder length $(n)$ and number of halfwaves along cylinder circumference $(2 m)$ reads [26]

$$
q_{2}(n, m)=\frac{\left\{\left(1-v^{2}\right) \lambda^{4}+k\left[\left(\lambda^{2}+\nu^{2}\right)^{4}-2\left(\nu \lambda^{6}+3 \lambda^{4} m^{2}+(4-v) \lambda^{2} m^{4}+m^{6}\right)+2(2-\nu) \lambda^{2} m^{2}+m^{4}\right]\right\}}{\lambda^{2}\left(\lambda^{2}+m^{2}\right)^{2}+\lambda^{2} m^{2}} .
$$

In the above formula the parameter $\lambda$ depends on value of $n$ and cylinder geometry: $\lambda=n \pi r / l$, while $k$ depends solely on thickness of cylinder wall and its radius: $k=t^{2} /\left(12 r^{2}\right)$. Minimal value of nondimensional loading parameter $q_{2}$ can be determined by minimisation of (10) over parameters $n$ and $m$ :

find $\{n, m\}$ such that $q_{2}(n, m)$ is minimal; $m, n$ are integers. 


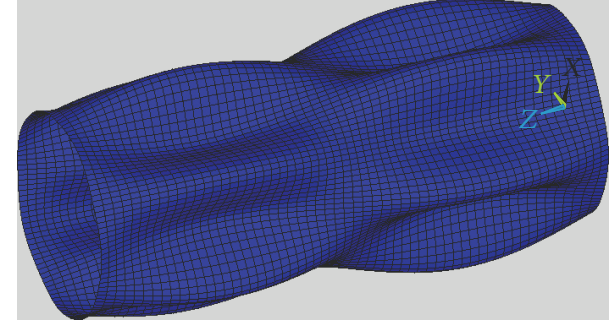

(a)

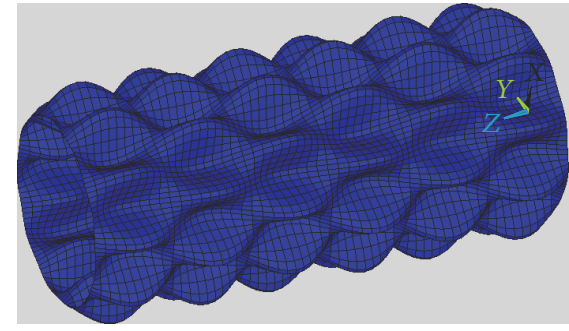

(b)

FIGURE 8: Buckling shapes of the cylindrical shell under axial loading (no internal pressure): (a) the second mode, (b) the fourth mode.

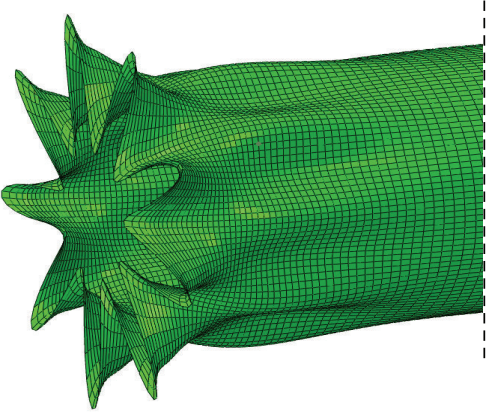

(a)

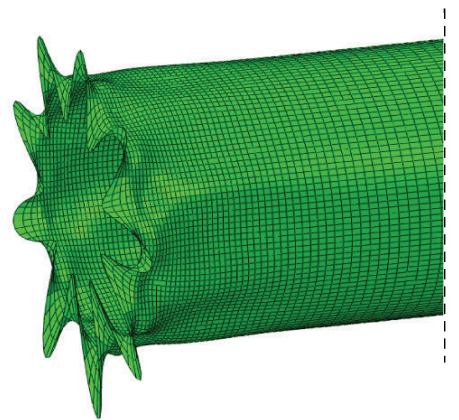

(b)

Figure 9: Buckling shapes obtained for internal pressure equal to (a) $p=0 \mathrm{MPa}$, (b) $p=0,07 \mathrm{MPa}$.

Minimum is usually obtained for $n=1$ and therefore minimisation over $m$ is sufficient for finding the critical value of parameter $q_{2}$. Further, the value of critical axial force which causes buckling of the cylinder can be calculated as

$$
F=2 \pi r D q_{2}(n, m),
$$

where $D=E t /\left(1-v^{2}\right)$ is in-plane stiffness.

The above described problem of buckling during axial compression was simulated with the use of finite element method in order to check the conformity of analytical and numerical approach. Linear analysis of buckling caused by axial load comprises solution of two-step problem defined by (7a), (7b) in which $\mathbf{Q}_{\mathrm{F}}$ indicates axial loading applied at free end of the cylinder and uniformly distributed along its circumference. The problem differs from the previously considered buckling under action of the bending force only by the direction of the applied loading $\mathbf{Q}_{\mathbf{F}}$ and physical sense of the load stiffness matrix $\mathbf{K}_{\mathrm{Q}_{\mathrm{F}}}$.

The buckling shapes obtained with the use of finite element method (Figures 8(a) and 8(b)) were characterised by sinusoidal weaving patterns predicted by the analytical approach. Moreover, the value of the first critical force as well as the values of critical forces corresponding to subsequent buckling modes computed numerically appeared to be in a very good agreement with analytical results obtained from (12).

The following computations were performed for the case of distributed loading applied perpendicularly at cylinder sidewalls modelling internal pressure of gas and the case of simultaneous action of axial loading and pressure. FEMbased simulations had fully confirmed the analytical results and conclusions drawn by Flugge. Internal pressure was found not to substantially change the value of critical compressive axial force; however the axial tension appeared to increase the resistance to buckling caused by an external pressure.

The next group of simulations was related to the lessexamined-in-the-literature situation when one end of the cylinder is clamped and axial loading is applied at the other end where no kinematic constraints are imposed. In such a case, the lack of symmetry of the loading and boundary conditions causes that analysis of buckling cannot be performed analytically and thus numerical approach has to be applied. The conducted numerical analyses reveal that buckling of the cylinder has rather local character and it is concentrated in the vicinity of the free end of the cylinder, Figure 9(a).

In general, the applied internal pressure causes stiffening of the cylindrical shell and its influence is reflected both in the buckling shapes and in the value of the critical force. Even for low values of pressure, the buckling area steadily moves in the direction of free end and gradually occupies a smaller part of the cylinder; compare Figure 9(b). Eventually, at a certain value of pressure (in the considered case $0,08 \mathrm{MPa}$ ) buckling shape changes and does not contain characteristic weaving of the free end of the cylinder. The value of critical force increases in the range of pressure $0-0.08 \mathrm{MPa}$ and then remains at an almost constant value; see Table 2 . 
TABLE 2: Change of critical force of axially compressed cylinder in terms of internal pressure.

\begin{tabular}{|c|c|c|c|c|c|c|c|c|c|c|c|}
\hline Pressure $[\mathrm{MPa}]$ & 0 & 0,01 & 0,02 & 0,03 & 0,04 & 0,05 & 0,06 & 0,07 & 0,08 & 0,09 & 0,1 \\
\hline Force $[\mathrm{N}]$ & 4071 & 4501 & 4729 & 4890 & 5002 & 5091 & 5159 & 5210 & 5236 & 5235 & 5231 \\
\hline
\end{tabular}

TABLE 3: Change of frequency of vibration $[\mathrm{Hz}]$ caused by internal pressure $[\mathrm{MPa}]$.

\begin{tabular}{lcccc}
\hline & $p=0$ & $p=0,2$ & $p=0,4$ & $p=0,6$ \\
\hline Mode I & 830,25 & 2556,0 & 2959,3 & 3189,9 \\
Mode II & 880,67 & 2709,3 & 3297,6 & 3900,8 \\
Mode III & 1005,6 & 3083,8 & 4243,7 & 5133,1 \\
Mode IV & 1079,8 & 3809,7 & 4670,9 & 5148,8 \\
\hline
\end{tabular}

3.5. Modal Analysis of Empty and Inflated Can. Modal analysis of empty and inflated can was performed in order to investigate change of basic dynamic characteristics caused by internal pressure. The modal analysis of the inflatable structure is preceded by the initial step when distributed loading modelling gas pressure is applied. Similarly as in the case of buckling analysis the initial prestressing step can be executed as a geometrically linear or nonlinear one; however only the second, more general case will be presented here for the sake of brevity. This preliminary stage of analysis is aimed at obtaining prestressed and deformed configuration of the structure which serves as a starting point for classical modal analysis performed at the second stage. The corresponding mathematical formulation of the problem reads as follows:

$$
\begin{array}{r}
\text { Step 1: } \mathbf{K}\left(\mathbf{Q}_{\mathbf{p}}, \mathbf{q}_{\mathbf{p}}\right) \mathbf{q}_{\mathbf{p}}=\mathbf{Q}_{\mathbf{p}}, \\
\text { Step 2: }\left[\mathbf{K}\left(\mathbf{Q}_{\mathbf{p}}^{\max }, \mathbf{q}_{\mathbf{p}}\right)-\omega^{2} \mathbf{M}\right] \boldsymbol{\varphi}=\mathbf{0},
\end{array}
$$

where $\omega$ is a natural frequency of the system and $\varphi$ indicates subsequent modes of vibration. Initial prestress of the structure by internal pressure influences stiffness matrix used in modal analysis. Consequently, initial prestress affects natural frequencies and modal shapes of the inflated structure determined from (13b).

Since the most significant effect of internal pressure is a uniform tension of the structure, the inflation causes that frequencies of vibrations increase. The effect is significant even if gas inside the cylinder is under low pressure (see Table 3) and it corresponds to subsequent vibration modes of the structure. Change of the vibration frequency is often associated with change of the modal shape, which in case of inflated structure involves smaller number of sinusoidal patterns in the circumferential direction, Figure 10. Let us note that two characteristic features of the structure considered within this section, that is, the buckling force and the frequency of vibration, are not independent of each other since they are connected by the dynamic criterion of the stability loss. More detailed studies on vibrations of cylindrical shells filled with fluid or exposed to fluid flow are presented in $[27,28]$.
The final conclusion from basic experiment and simulations conducted in two previous sections is that application of internal pressure substantially changes the state of stress in the structure, the value of the buckling force, and the frequencies of structure vibrations. Thus, basic mechanical properties and dynamic characteristics of thin-walled structures can be conveniently and efficiently modified with the use of internal pressure.

\section{Analysis of Selected Applications of Inflatable Thin-Walled Structures}

The following section presents numerical simulations of the concepts of several types of thin-walled structures subjected to impact loading and attempt of improvement of their crash properties by using inflation with compressed gas. The following concepts of engineering structures will be examined:

(i) inflatable compartments inside hull of the ship,

(ii) inflatable door of the passenger car,

(iii) inflatable traffic barrier.

In all the above inflatable structures a gas pressure will be maintained on a constant level during the entire impact process (e.g., with the use of automatic check valves). Such approach allows avoiding uncontrolled pressure increase, which could easily lead to ductile failure and bursting of the structure. For each structure the influence of internal pressure on global durability and energy absorption capabilities will be analysed. The range of required pressure values will be estimated and benefits of dividing the structure into several separate chambers with separately adjusted pressure levels will be studied.

Modelling of inflatable structures subjected to impact loading is a coupled problem, which requires combination of finite element-based modelling of the structure and thermodynamic modelling of the gas. In basic thermodynamic approach the gas is typically modelled with the use of the so-called uniform pressure method, which assumes that gas pressure, temperature, and density are uniform within a single gas chamber. Thus, in general case of inflatable structure equipped with inflow/outflow devices the additional unknowns of the coupled problem are mass of the gas, its pressure, and temperature. Change of these parameters is defined by three equations: differential equation governing the balance of fluid mass, differential equation governing the balance of fluid internal energy, and (typically algebraic) equation of state. Coupling between the structure and the fluid is provided by the kinematic coupling condition, which ensures the equality of actual gas volume and chamber volume, as well as the force coupling condition, which 

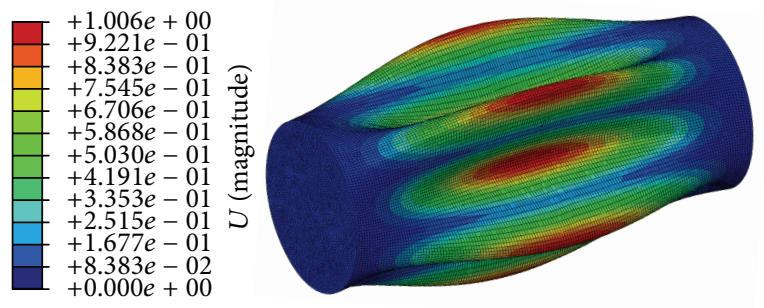

(a)

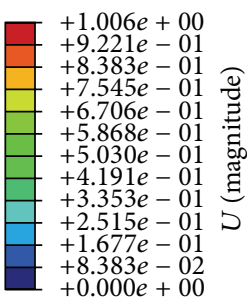

$+0.000 e+00$

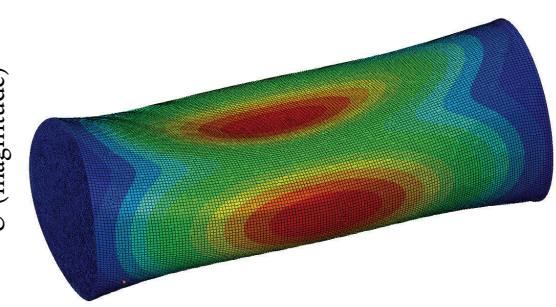

(b)

FIGURE 10: Change of first vibration mode of the cylinder caused by internal pressure: (a) $p=0 \mathrm{MPa}$, (b) $p=0,6 \mathrm{MPa}$.

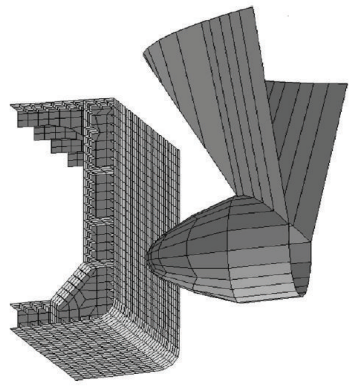

(a)

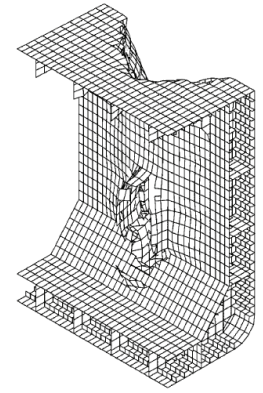

(b)

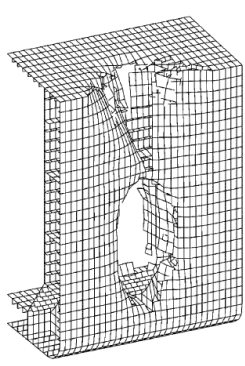

Figure 11: Collision of double-hull carrier and ship with a bulbous bow: (a) numerical model of the problem, (b) typical damage of the struck ship [12].

indicates that pressure of gas constitutes external loading for the structure $[29,30]$.

The substantial simplification of the above coupled model results from the fact that in considered structures the level of applied pressure is maintained constant. In such case a general model of inflatable structure is decoupled into two parts. The initial stage of solution utilises exclusively finite element model of the structure and additional loading modelling pressure exerted by compressed gas. It allows computing deformation of the structure and change of volume of internal gas. In the second stage of solution the balance of fluid mass, the balance of fluid energy, and the equation of state can be used to compute change of fluid mass, change of fluid temperature, and the required opening of the valve. Since presented examples are aimed at investigation of the global structural response of inflatable structure, the numerical simulations will involve exclusively the first stage of the above solution.

The solver used for all numerical simulations included in this section was commercial finite element code ABAQUS Explicit while the main finite element applied in simulations was doubly curved thin shell element S4R. The choice of explicit method of integration of the equations of motion allowed eliminating convergence difficulties typically encountered in implicit methods. Thus, it enabled obtaining robust solution of considered strongly nonlinear impact problems.
4.1. Inflatable Hull Compartments. The first of the analysed concepts is inflation of the compartments located inside the hull of the ship in order to increase hull durability and to prevent its total penetration in case of open-sea ship collisions.

The problem of ship collisions was extensively analysed in the literature with the use of both analytical [31] and numerical approaches [32]. According to the conducted studies one of the most dangerous ship collision scenarios is impact of a ship with a bulbous bow against double-hull carrier (Figure 11(a)). The systematic numerical simulation of such collision was performed, for example, by Haris and Amdahl [33] as well as Wiśniewski and Kołakowski [12]. In the latter paper the hull of the struck ship was composed of inner and outer hull layer, stiffeners, and hull plating, while the bulbous bow of the striking ship was assumed to be a rigid body. The struck ship was still, and the striking ship was moving in horizontal direction such that collision occurred at right angle. The results of numerical simulations indicated that typical damage of the struck ship caused by impact of moderate velocities involves total penetration of the carrier hull (Figure 11(b)).

The preliminary verification of the concept of the inflation of hull compartments will be examined by using simple numerical example in which a cuboid of dimensions $6 \mathrm{~m}$ $\times 4 \mathrm{~m} \times 1,5 \mathrm{~m}$ and thickness of $0,015 \mathrm{~m}$, which resembles a single compartment of the ship hull, is impacted by rigid 

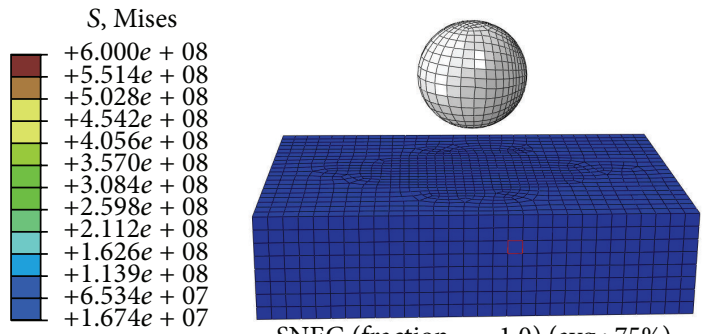

SNEG (fraction $=-1.0)($ avg.: $75 \%)$

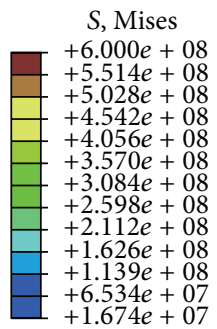

(a)

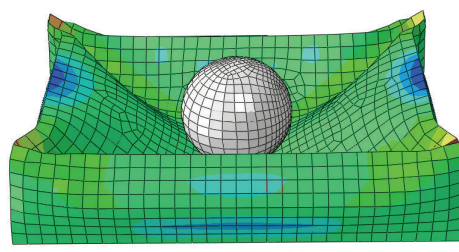

SNEG $($ fraction $=-1.0)($ avg.: $75 \%)$

(c)

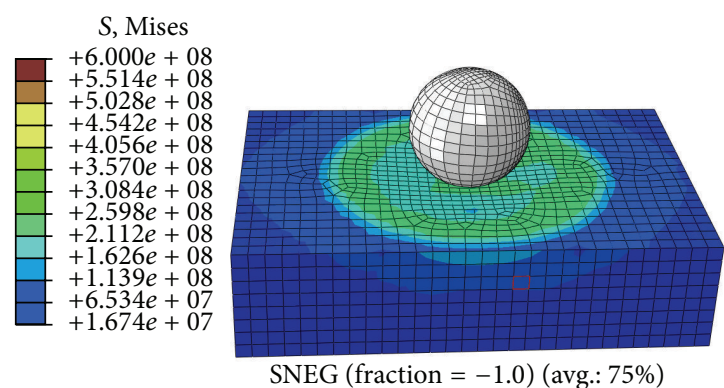

(b)

$S$, Mises

$+6.000 e+08$ $+5.514 e+08$ $+5.028 e+08$ $+4.542 e+08$ $+4.056 e+08$ $+3.570 e+08$ $+3.084 e+08$ $+2.598 e+08$ $+2.112 e+08$ $+1.626 e+08$ $+1.139 e+08$ $+6.534 e+07$
$+1.674 e+07$

$+1.674 e+07$

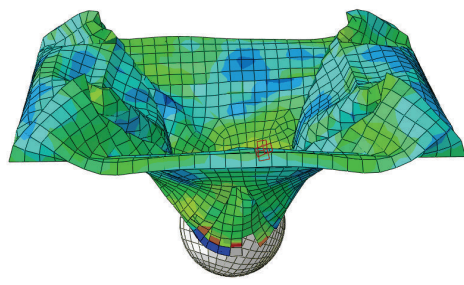

SNEG (fraction $=-1.0)($ avg.: $75 \%)$

(d)

FIGURE 12: Simulation of impact against empty compartment ( $M=800$ tons, $V=10 \mathrm{~m} / \mathrm{s})$ : (a) initial configuration, (b) preliminary stage of impact, (c) intermediate stage of impact, and (d) full penetration of the compartment.

sphere of diameter $1,6 \mathrm{~m}$, which resembles a bulbous bow (Figure 12). The proposed simplified simulation is based on the previously mentioned simulation of ship collision in terms of basic dimensions and material properties. The characteristics of the compartment material were assumed as elastoplastic with hardening. Tensile failure of the material occurs when ultimate plastic strain $(\varepsilon=0,17$ at $\sigma=400 \mathrm{MPa})$ is exceeded in all Gauss points of the element. Although the proposed numerical model does not directly correspond to the case of ship collision, which would require more accurate representation of larger parts of both colliding ships, it is expected to provide very basic and rough verification of the proposed concept of hull inflation.

In the initial simulation, empty compartment was subjected to impact characterised by fixed velocity equal to $10 \mathrm{~m} / \mathrm{s}$ but various masses of the striking object. Due to partiality of the model two types of kinematic boundary conditions were considered:

(i) suppression of displacements of the lower edge of the compartment,

(ii) suppression of displacements of both the upper and the lower edge.

Since deformation of the compartment significantly differs in both cases, the process of impact and the influence of inflation will be analysed separately for each structure.

In the first case, when the edge of the upper surface remains unconstrained, both upper and lower surface of the chamber are simultaneously involved in the process of impact absorption. After the beginning of impact, the upper surface deforms until the contact with the lower surface occurs. In the following stage, both surfaces remain in contact and they deform together. The lower surface usually ruptures first as a result of kinematic boundary conditions applied along all its edges. Finally, the upper surface of the chamber also bursts and total penetration by the hitting object occurs. In the considered example, all stages of the process take place for the impacting mass of 800 tons and above, Figure 12. The numerical simulation conducted for such impact loading indicates that the structure dissipates $90,5 \%$ of the kinetic energy of impacting object during the entire process of impact.

The beneficial influence of chamber inflation is most transparent during the first stage of impact when internal pressure increases resistance of the upper surface against impact loading. In the second stage of impact when both surfaces come into contact, the advantageous effect of inflation relies on increase of global stiffness of the compartment. At the time instant when lower surface is ruptured, the influence of internal pressure is totally suppressed due to immediate pressure release.

In the presented numerical example (Figure 13), the impact loading is exactly the same as previously and internal pressure is maintained at the lowest constant level for which a qualitative difference in structural response is observed ( $p=0,4 \mathrm{MPa}$ ). Applied internal pressure allows avoiding rupture of the upper surface and thus it prevents full penetration of the compartment by impacting object. The inflated compartment is able to dissipate $100 \%$ of the initial kinetic energy of the impacting object. Thus, the increase in energy dissipation capability caused by initial inflation equals at least 9,5\%. Moreover, substantially higher value of constant 
$S$, Mises

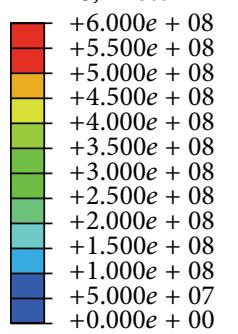

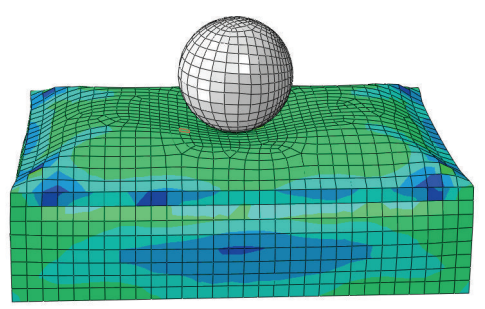

SNEG (fraction $=-1.0)($ avg.: $75 \%)$

(a)
$S$, Mises

$+6.000 e+08$

$+5.500 e+08$

$+5.000 e+08$

$+4.500 e+08$

$+4.000 e+08$

$+3.500 e+08$

$+3.000 e+08$

$+2.500 e+08$

$+2.000 e+08$

$+1.500 e+08$

$+1.000 e+08$

$+5.000 e+07$

$+0.000 e+00$

(b)

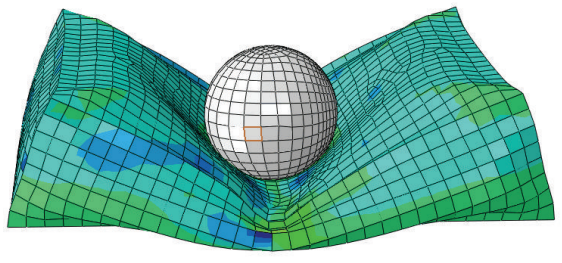

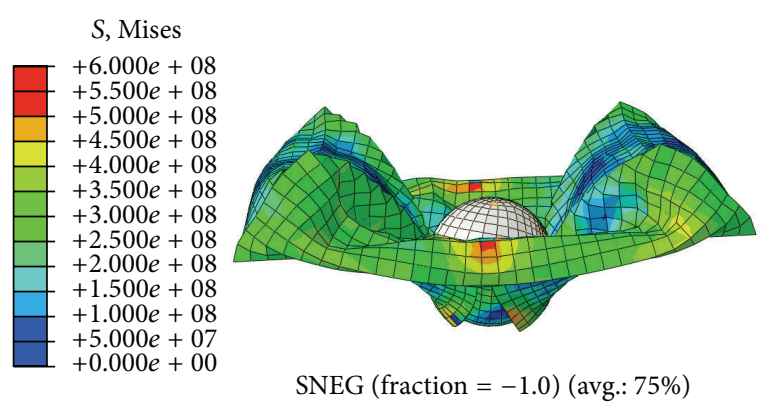

(c)

FIGURE 13: Simulation of impact against inflated compartment $(M=800$ tons, $V=10 \mathrm{~m} / \mathrm{s}$, and $p=0,4 \mathrm{MPa})$ : (a) initial stage of impact, (b) intermediate stage, and (c) final stage with rupture of the lower surface.

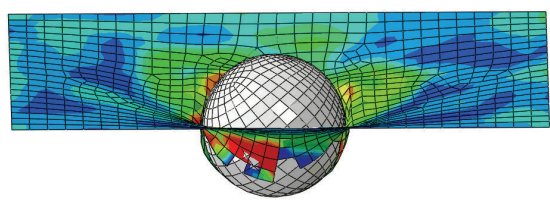

(a)

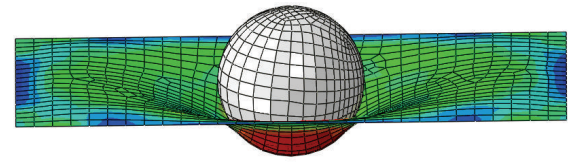

(b)

FIGURE 14: Simulation of the impact process $(M=150$ tons, $V=10 \mathrm{~m} / \mathrm{s})$ : (a) final deformation of an empty compartment, (b) final deformation of the inflated compartment.

internal pressure $(p>0,8 \mathrm{MPa})$ allows obtaining a different mode of deformation in which destruction of lower surface is prevented; however the upper surface bursts. Although none of the above strategies allows maintaining total structural integrity of the compartment, the presented example clearly indicates that internal pressure allows avoiding full penetration by impacting object and controlling the type of compartment failure.

In case of the second type of boundary conditions, when displacement of the upper edge of the compartment is also constrained, both surfaces of the cuboid respond almost independently. Kinematic constraints imposed on the upper surface do not allow for its large deflection and contact with the lower surface. Therefore, the impacting object is initially stopped exclusively by the upper surface and when it ruptures, exclusively by the lower surface, Figure 14(a). Mass of the object which causes penetration of the upper layer of noninflated structure is substantially lower than in previous case and equals 150 tons. According to the performed numerical simulation for such impact loading the upper surface of the compartment dissipates $75,3 \%$ of the kinetic energy of impacting object during the process.

Despite different operating principle and mechanical behaviour the internal pressure can still be used to improve durability of the structure against impact loading. In the considered case the pressure of gas will be applied to increase total force generated on the impacting object and to avoid rupture of the upper layer of the compartment. The inflation will help to improve the crashworthiness of the upper surface of the cuboid; however it will have no beneficial influence on the behaviour of the lower one.

In the presented numerical example internal pressure is maintained at a lowest constant level $(p=0,6 \mathrm{MPa})$ for which failure of the upper surface does not occur. Nevertheless, the strategy results in slight rebound of the impacting body and allows preserving global integrity of the compartment, 


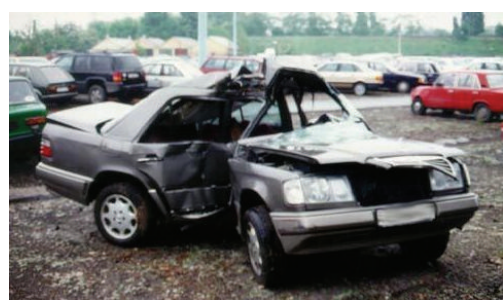

(a)

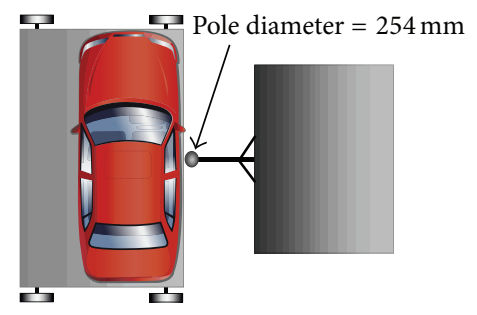

(b)

FIGURE 15: Lateral impact against door of the passenger car: (a) deformation after impact with high velocity, (b) scenario of NCAP "pole side impact" test.
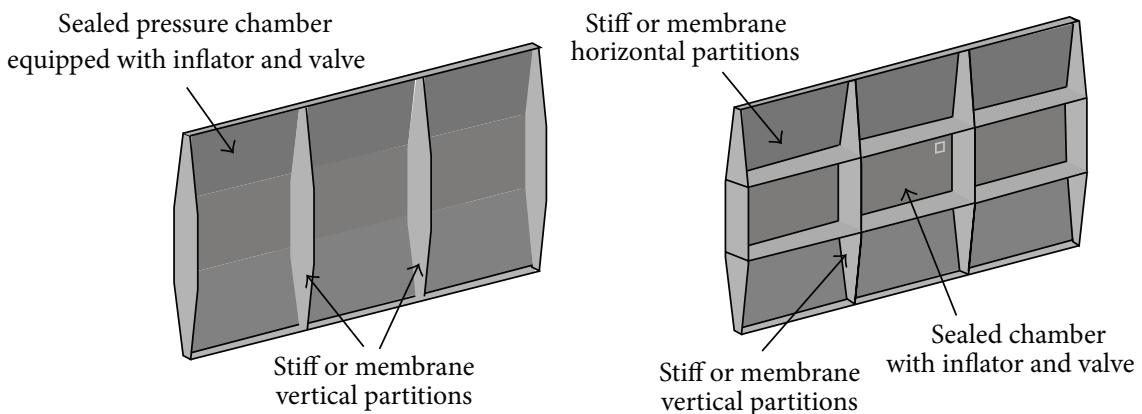

Figure 16: The concept of multichamber inflatable door of the passenger car.
Figure 14(b). Since the inflated compartment is able to dissipate total initial kinetic energy of the impacting object the increase in energy dissipation capability of the inflated compartment is around $25 \%$.

4.2. Inflatable Door of the Car. The second proposed concept is inflation of the door of the passenger car in order to increase its durability in case of lateral impact against narrow objects. Lateral collision with objects such as pole, lantern, or tree is a typical accident scenario and in case of high impact velocities may cause severe destruction of the entire body of the car, Figure 15(a). Lateral impact is also included as a mandatory crash-test for each produced car according to the European New Car Assessment Programme (Euro-NCAP). In this test, the car is propelled sideways at $29 \mathrm{kph}$ into a rigid pole of a diameter 25,4 mm, Figure 15(b).

The concept of inflatable door of the car is based on vertical, horizontal, or both-directional division of its internal space into sealed pressure chambers (Figure 16). Each chamber is assumed to be inflated to different initial pressure and to be equipped with separate devices allowing maintaining constant pressure level. The chambers can be constructed with the use of stiff partitions made of the same material as the door itself or, alternatively, by flexible membrane partitions. In the case when the partitions are stiff they substantially increase durability of the door against side impact. Therefore, the analysis of the effectiveness of inflation has to be performed by comparing empty and inflated structure of the same topology.

Numerical simulations within this section are based on NCAP "pole side impact" test. Nevertheless, the kinematics of the system is reversed: the immobile door of the car (dimensions: $1 \mathrm{~m} \times 0,6 \mathrm{~m} \times 0,1 \mathrm{~m}$, thickness $0,001 \mathrm{~m}$ ) supported in out-of-plane direction on the lateral edges is subjected to impact of a rigid pole of fixed mass and velocity $(M=$ $1000 \mathrm{~kg}$ and $V=4 \mathrm{~m} / \mathrm{s}$ and $V=8 \mathrm{~m} / \mathrm{s}$ ), Figures 17 and 18 . The main requirement for the door of the car is to minimise deformation caused by the hitting object in order to protect passenger's area. Consequently, the main quantity observed in each simulation is maximal deflection caused by pole impact. Let us however note that deformation of the door strongly depends on kinematic constraints imposed on inplane displacement of its lateral edges. Thus, two possible types of kinematic boundary conditions (free and blocked) will be considered separately.

The first numerical example concerns door with three vertical chambers and fixed in-plane displacement of the lateral edges, subjected to impact with the velocity of $8 \mathrm{~m} / \mathrm{s}$. Impact against empty structure results in substantial deformation with maximal displacement of the central part equal to $0,22 \mathrm{~m}$, Figure $17(\mathrm{a})$. The simple strategy of inflation, proposed in order to improve response of the structure, assumes twice higher initial pressure in impact-subjected central chamber and maintaining constant level of pressure in all chambers during the impact process. The application of the above strategy (with pressures $0,25 \mathrm{MPa}, 0,5 \mathrm{MPa}$, and $0,25 \mathrm{MPa}$ ) results in a substantial change of deformation shape and decrease of maximal displacement to $0,17 \mathrm{~m}$, Figure 17(b). The effect of inflation is also clearly visible after rebound of the impacting object. After impact the noninflated structure remains fully deflected by impacting object while inflated structure partially retains its initial 

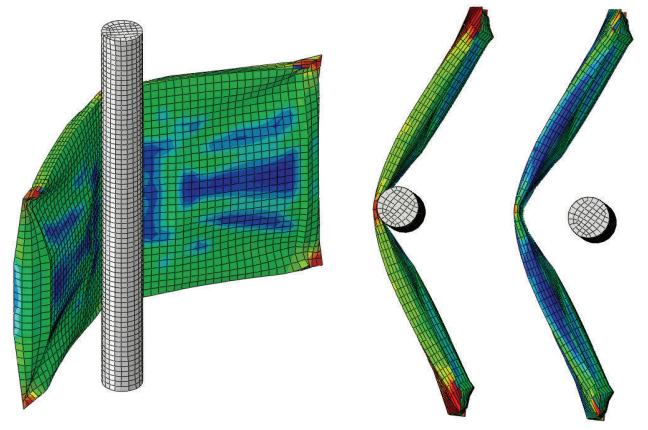

(a)
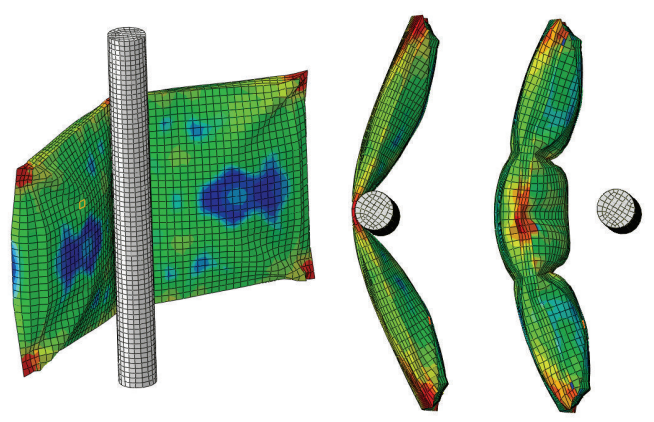

(b)

FiguRE 17: Response of three-chamber structure to lateral impact $(M=1000 \mathrm{~kg}, V=8 \mathrm{~m} / \mathrm{s}$, suppressed in-plane displacement): (a) no inflation, (b) constant pressure: $0,25 \mathrm{MPa}, 0,5 \mathrm{MPa}$, and 0,25 $\mathrm{MPa}$.

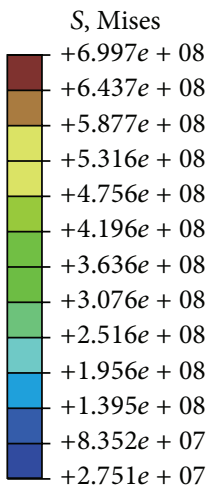

SNEG (fraction $=-1.0)($ avg.: $75 \%)$

(a)
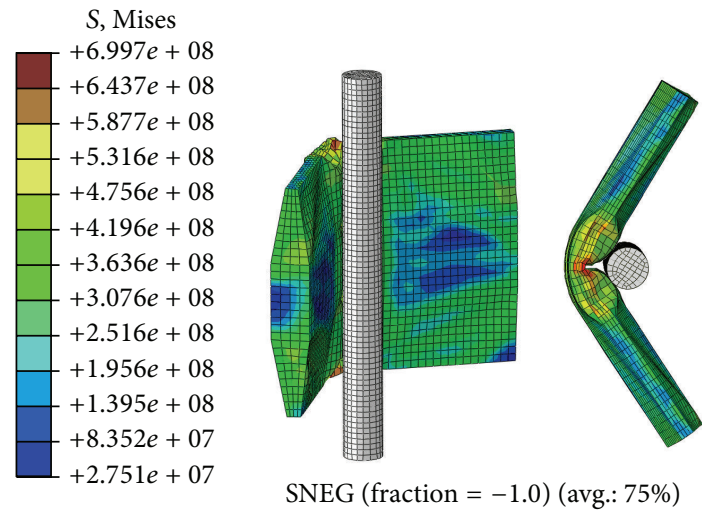

SNEG $($ fraction $=-1.0)($ avg.: $75 \%)$

(b)

FIGURE 18: Response of nine-chamber structure on lateral impact ( $M=1000 \mathrm{~kg}, V=4 \mathrm{~m} / \mathrm{s}$, free in-plane displacement): (a) no inflation, (b) constant pressure of 0,5 $\mathrm{MPa}$.

configuration simultaneously remaining bulged by internal pressure, Figure 17(b).

The influence of inflation is even more apparent in case of structure with free in-plane displacement of the lateral edges (free-supported structure). In numerical simulation the structure divided into nine pressure chambers was subjected to impact with initial velocity equal to $4 \mathrm{~m} / \mathrm{s}$. Despite larger initial stiffness of the structure and smaller impact energy the deformation of the structure is larger than in previous case (max. displacement: $0,38 \mathrm{~m}$ ). Stopping of the impacting object is the result of increasing resistance force caused by change of the bended beam direction and it lasts until clamping of the pole, Figure 18(a). In turn, the inflation of the structure with uniform pressure of $0,5 \mathrm{MPa}$ allows substantially changing the shape of structure deformation (Figure 18(b)) and decreasing its maximal deflection by approx. $40 \%$ (to $0,23 \mathrm{~m}$ ).

The above simulations were supplemented with the analysis of the contact force acting between the pole and the structure. The change of contact force in standard and inflated door is presented in terms of impact time and displacement of the impacting pole in Figures 19(a) and 19(b), respectively.
The comparison of plots of both contact forces evidently indicates the benefits resulting from structure inflation. In case of standard structure the change of contact force is clearly nonoptimal. The initial peak of force followed by its substantial decline and reincrease at the end of the process. In turn, in case of inflated structure the generated contact force is more steady; the initial peak of force is followed by its slow gradual decrease until the end of the process. Higher average value of contact force in terms of displacement allows stopping the impacting object by using a shorter distance.

Integration of the contact force presented in Figure 19(a) over impact time allows obtaining change of impacting object momentum during the process $\Delta \mathbf{p}$. The change of momentum is slightly larger in case of inflated structure, which corresponds to larger rebound velocity of the impacting object. In turn, integration of the contact force presented in Figure 19(b) over displacement allows calculating energy dissipation and corresponding change of kinetic energy of the impacting body $\Delta E$. In case of both the empty and inflated structure the point of maximal displacement corresponds to maximal change of kinetic energy ( $\Delta E=8000 \mathrm{~J})$ being equal to its initial value. However, in case of inflated structure the action 


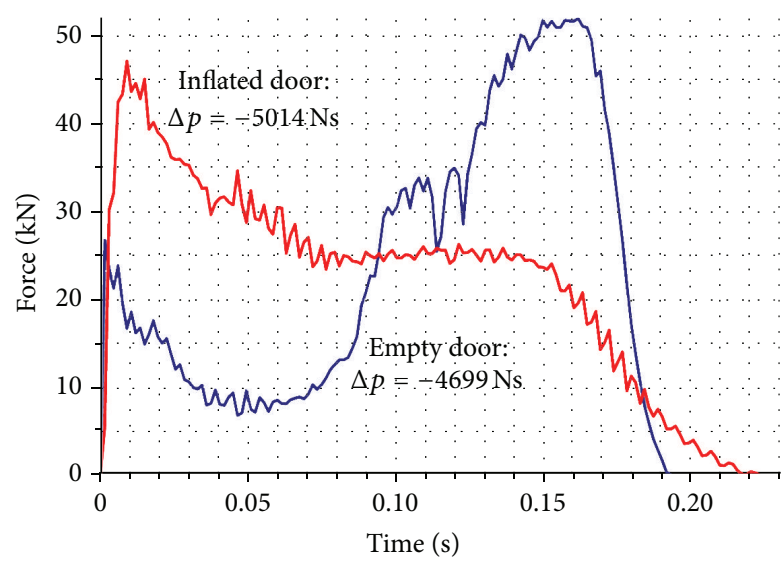

(a)

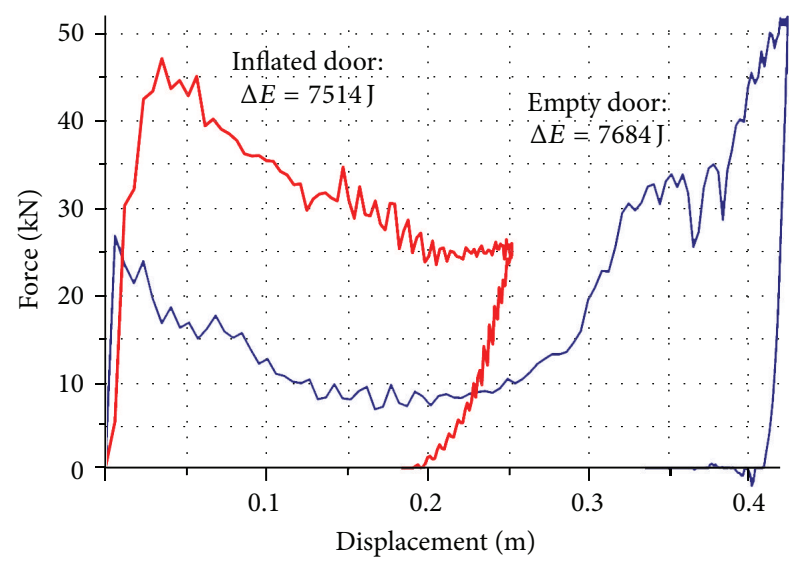

(b)

FIGURE 19: Contact force generated during collision in standard and inflated structure: (a) force versus time, (b) force versus pole displacement.

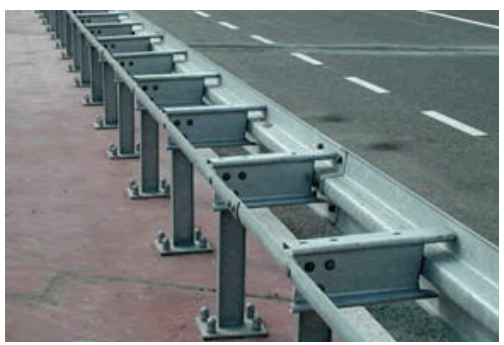

(a)

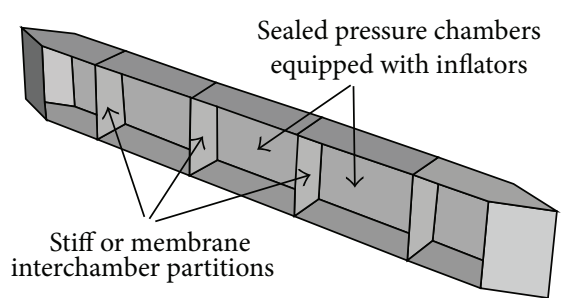

(b)

FIGURE 20: Comparison of two various designs of a traffic barrier: (a) standard guardrail design, (b) the concept of inflatable road barrier.

of compressed gas causes that more energy is returned back to impacting object and it rebounds with a slightly larger velocity.

Both examples presented in this section indicate that application of internal pressure allows increasing global stiffness of thin-walled structure and, consequently, considerably diminishing the deformation caused by impacting object.

4.3. Inflatable Traffic Barrier. The last considered concept of inflatable thin-walled structure is a multichamber inflatable traffic barrier, Figure 20(a). Traffic barriers prevent errant vehicles from falling out of the road and being subjected to obstacles or hazards located nearby. They can be divided into three groups depending on their stiffness:

(i) flexible barriers (as metal beam fence, cable barriers, and corrugated rail systems),

(ii) semirigid barriers (as standard guardrail barrier described below),

(iii) rigid barriers (as block of reinforced concrete).

Standard guardrail barriers are constructed of sigma posts, distance spacer, and a guardrail, Figure 20(a). They dissipate energy of impact by deformation of barrier components, friction between barrier and the car, and deformation of the car's body. Semirigid barriers usually allow for deformation up to $1 \mathrm{~m}$ in case of the harshest impact. According to code regulations, the barriers are designed to sustain impact of heavy vehicle with large initial velocity but simultaneously to provide an acceptably low level of deceleration of light vehicles. Since the above requirements are contradictory, a typical barrier cannot fulfil both of them in an optimal way.

Due to the above reason in addition to standard design several innovative concepts of traffic barriers were proposed. One of them is based on a cable net equipped with hydraulic absorbers on both sides, which allow controlling the force used for stopping the car. Another design utilises the panel embedded in surface of the road, which after activation by a sensor extends above the road and stops the vehicle [34]. The idea of inflatable traffic barrier divided into pressure chambers was proposed by the authors in [24] and the similar concept of barrier equipped with internal airbags was later analysed in [35].

Here, the potential of application of compressed gas inside thin-walled road barrier will be examined with the use of conceptual design presented in Figure 20(b). In numerical simulations, the barrier of dimensions $3 \mathrm{~m} \times 0,2 \mathrm{~m}$ $\times 0,4 \mathrm{~m}$ and material thickness of $3 \mathrm{~mm}$ was subjected to perpendicular and skew (45 degrees) impact of the rigid body of dimensions of the car, mass $1000 \mathrm{~kg}$ and velocity of $16 \mathrm{~m} / \mathrm{s}$. The presented results concern three-chamber inflatable barrier with steel partitions of thickness $1 \mathrm{~mm}$. 

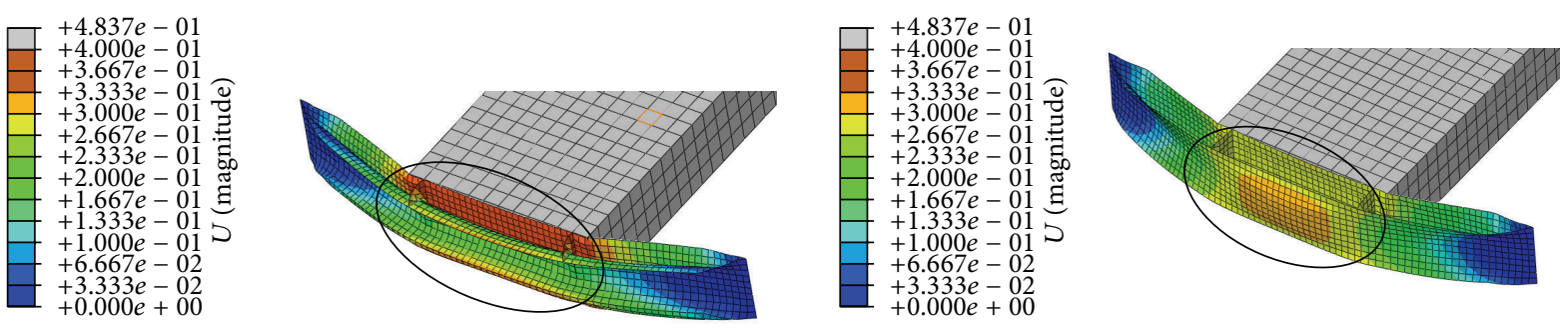

(a)
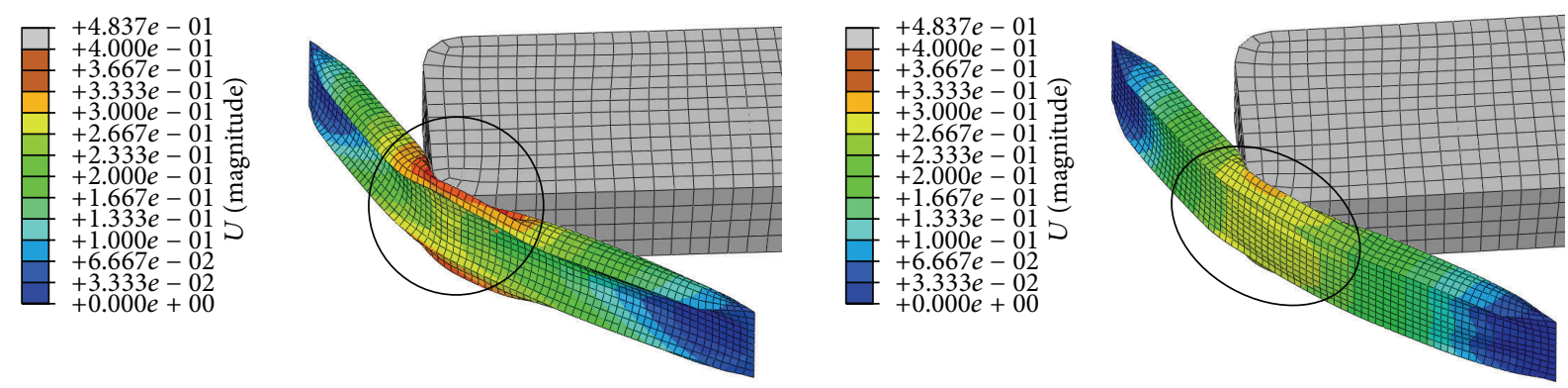

(b)

FIGURE 21: Comparison of the responses of empty barrier (left) and inflated barrier (right) to perpendicular impact (a) and skew impact (b).

In the case when the barrier is not inflated the perpendicular impact causes folding of the upper wall, crushing of the internal partitions, and substantial reduction of barrier width during the process, Figure 21(a) (left). Applied inflation of the barrier (constant pressures of $0,3 \mathrm{MPa}, 0,5 \mathrm{MPa}$, and $3 \mathrm{MPa}$ in subsequent chambers) increases local stiffness of each chamber and prevents crushing of the internal partitions, Figure 21(a) (right). Such effect has the influence on both local and global response of the barrier. At first, the barrier preserves its original width and distribution of local stresses beneficially changes. Secondly, the global stiffness of the barrier increases and the way of stopping the impacting object is reduced (from $0,34 \mathrm{~m}$ to $0,28 \mathrm{~m}$ ). In case of skew impact (Figure 21(b)) the internal pressure has similar positive effect. It results in reduction of local stress concentration at impact location and increase of the global stiffness of the barrier.

The above examples show that inflation of the particular internal chambers of thin-walled structure allows modifying its global stiffness and, moreover, positively influencing the zones of local stress concentration.

\section{The Concept of Adaptive Inflatable Barrier}

Adaptive inflatable thin-walled barriers can be also considered as one of the special technologies for adaptive impact absorption [36]. In contrast to passive inflatable structures, their operation is based not only on effective use of compressed gas, but also on real-time adjustment of initial inflation and precise control of internal pressure during the impact process. Such operating principle enables adaptation to actual impact loading and optimal dissipation of the corresponding impact energy. The concept of adaptive inflatable thin-walled barriers follows a more general concept of adaptive inflatable structures [29], which embraces adaptive pneumatic cylinders [37], inflatable fenders for protecting offshore towers [38], and external airbags for emergency landing.

In the proposed adaptive inflatable barrier each internal pressure chamber is equipped with fast inflator and controllable high-performance discharge valve, Figure 22. Assumed operation of the system comprises the initial stage of impact detection of identification (e.g., by ultrasonic sensors or dedicated "impactometer sensor" [39]) and the stage of adaptation to impact loading, which is composed of two separate steps. At the very beginning of the impact, the embedded inflators are triggered in order to inflate each chamber to appropriate initial pressure, which provides desired initial stiffness of the barrier. During impact the discharge valves mounted in internal partitions and external walls of the barrier control the flow of gas between the chambers and its outflow to environment. As a result, change of pressure in particular parts of the inflatable structure can be precisely controlled and, moreover, actual global stiffness and total resistance force can be continuously modified and adjusted to actual level of loading. The proposed method allows enhancing the process of energy dissipation and achieving desired dynamic response of the impacting object.

5.1. The Simplified Model of the Barrier. Miscellaneous strategies of pressure control will be developed for a twodimensional frame structure (Figure 22) which can be considered as a very basic model of introduced thin-walled barrier. This model of inflatable structure was selected due 


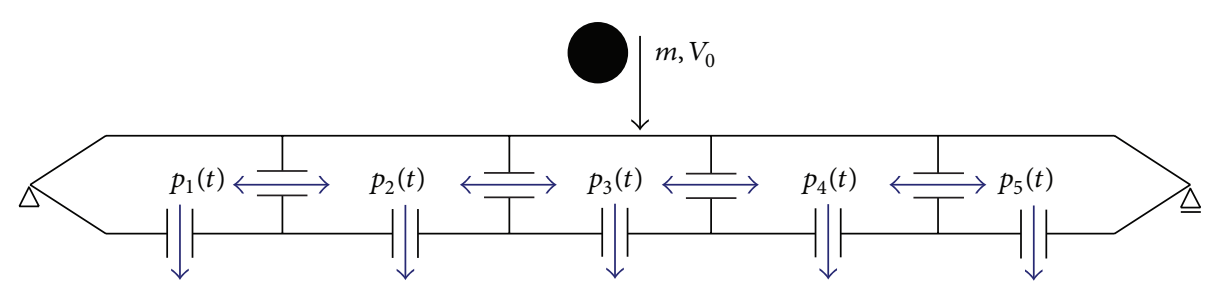

FIGURE 22: Two-dimensional frame structure with several separate pressure chambers considered in the optimisation and control problems.

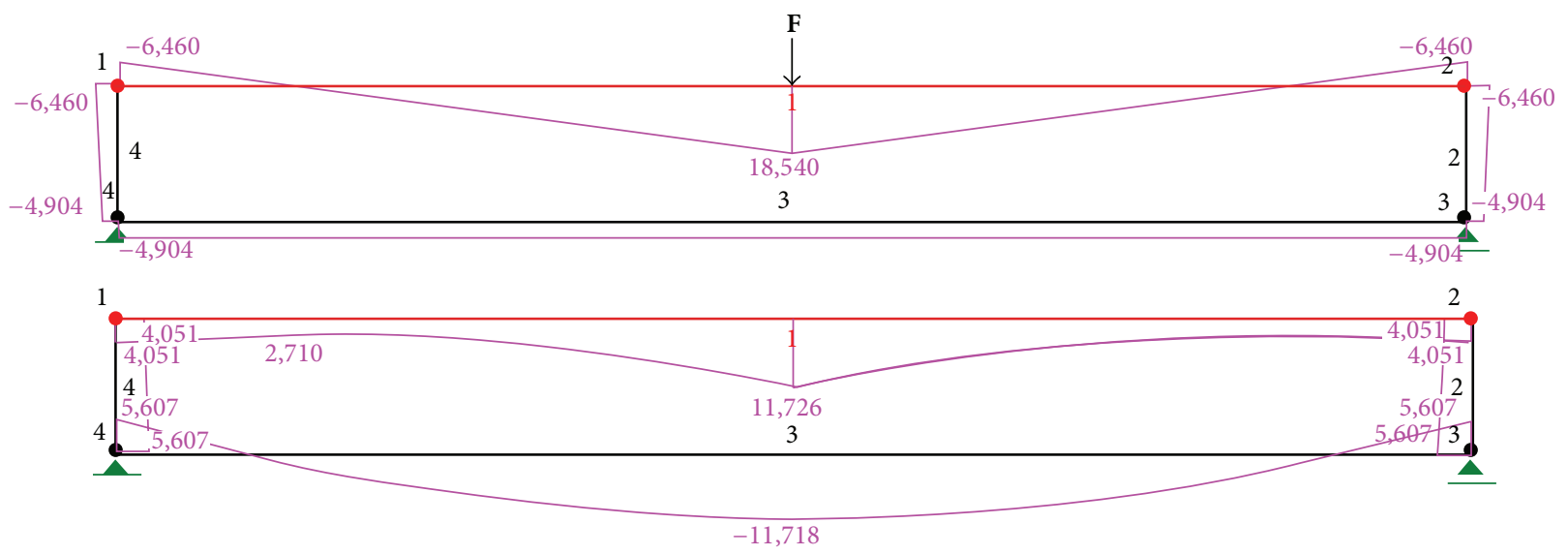

FIGURE 23: Reduction of bending moment obtained by optimal adjustment of internal pressure.

to its simplicity and high computational efficiency, which are required in solution of the considered optimisation and control problems. Obviously such a simple model does not possess all features of inflatable thin-walled traffic barriers and thus it cannot precisely imitate the entire process of energy dissipation and the corresponding crashworthiness characteristics. Nevertheless, it is expected that the proposed methodology of pressure driven control can be generalised and applied to three-dimensional thin-walled inflatable structures of arbitrary construction.

The frame structure under consideration may contain arbitrary number of pressure chambers separated by stiff or elastic partitions. The impact loading is modelled either by external force applied at the upper beam or by external object of a given mass and velocity. The model accounts for material nonlinearities (elastoplastic material model) and geometrical nonlinearities (equilibrium equations related to actual configuration). Each chamber of the frame is assumed to be equipped with inflators and controllable discharge valves which enable fast inflation and release of gas. Consequently, in contrast to passive inflatable structure the changes of pressures can be arbitrarily modified during the impact process in order to control global dynamic response of the system.

The considered control objectives were aimed at minimisation of internal forces in selected elements of the frame, minimisation of impacting object deceleration, and control of the final shape of structure deformation. In all cases the control was performed with respect to functions describing changes of pressures in particular chambers. Such approach allows decoupling general model of inflatable structure into structural part and thermodynamic part (cf. Section 4) and considering exclusively the structural model with distributed pressure loading.

5.2. Adjustment of Internal Pressure to Static Loading. The preliminary step before analysis of the impact loading and large deformations of the frame will be a linear static analysis aimed at investigation of influence of internal pressure on distribution of generalised internal forces in selected elements. Since the considered frame is statically indeterminate, the internal forces will be computed with the use of finite element method, which provides good approximation of the solution of considered linear static problem. The solution of global equilibrium equation $\mathbf{K q}=\mathbf{Q}$ allows determining local deformation of each element of the frame (longitudinal displacement $u(x)$ and deflection $w(x)$ ) and calculating normal forces $\mathbf{N}$, shear forces $\mathbf{T}$, and bending moments $\mathbf{M}$

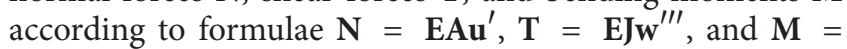
$\mathbf{E J w}^{\prime \prime}$, where $\mathbf{E}$ is a vector of Young moduli of frame elements and $\mathbf{A}$ is a vector of their cross-sectional areas while $\mathbf{J}$ is a vector of their moments of inertia. In the following examples only the value of bending moment will be considered since it corresponds to the largest values of generated stresses.

In the considered numerical example the frame which contains only one chamber is loaded in the middle of the upper span by a point load $\mathbf{F}$ of a unit magnitude. When the structure is not inflated the solution of the problem $\mathbf{K q}=$ $\mathbf{Q}_{\mathrm{F}}$ reveals a linear distribution of bending moments on the upper span with the largest bending moment in its middle point $\left(M_{\max }=18,54 \mathrm{kNm}\right)$, Figure 23 (top). In turn, when the frame is additionally inflated and subjected to the action of 


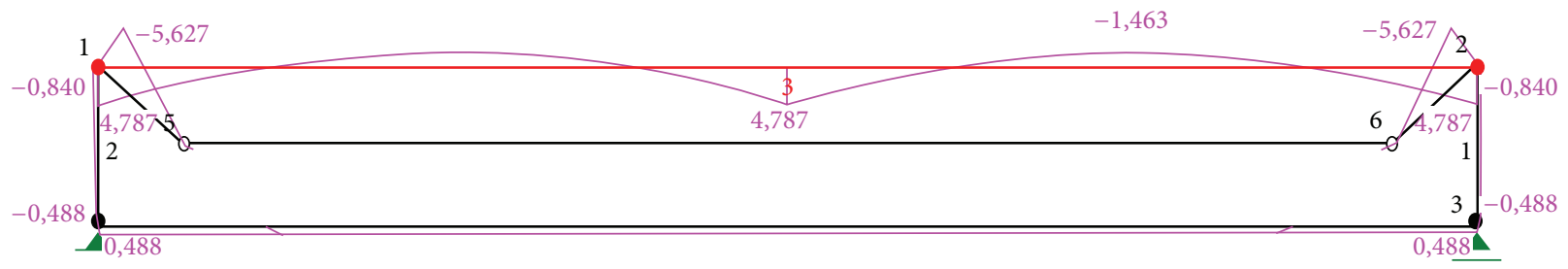

FIGURE 24: Reduction of bending moment obtained by horizontal division of the frame and inflation of the internal upper chamber.

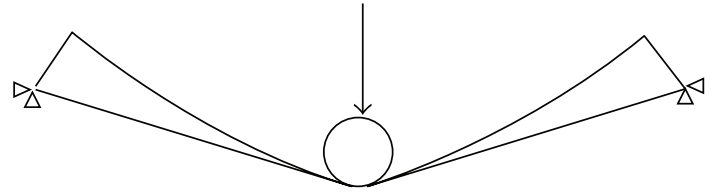

(a)

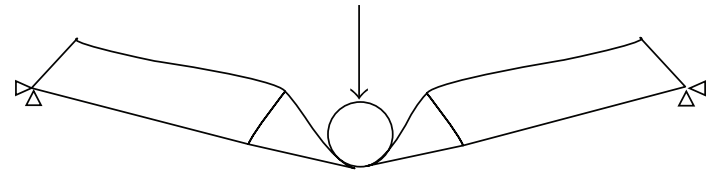

(b)

FIGURE 25: Inflatable structure considered in the problem of deceleration minimisation.

gas pressure the equilibrium is described by the following equation:

$$
\mathbf{K q}=\mathbf{Q}_{\mathbf{F}}+p \mathbf{Q}_{\mathbf{p}}
$$

where $\mathbf{Q}_{\mathbf{p}}$ is a load vector which models distributed loading of a unit value and $p$ is a scalar multiplier. Thus the problem of finding internal pressure, which minimises maximal value of bending moment, can be written in the following form:

$$
\text { find } p \text { such that } \max M(x) \text { is minimal }
$$

and $M(x)$ indicates bending moment resulting from the solution of (14). Since applied internal pressure causes reduction of bending moment at the upper beam but simultaneously increases bending moment at the lower beam, the optimal strategy assumes equalisation of both bending moments. The procedure of determination of the corresponding optimal pressure effectively utilises problem linearity. Application of the superposition principle allows calculating the value of additional bending moment which has to be generated by compressed gas and the corresponding value of required internal pressure.

The final solution of the above optimisation problem indicates that optimal value of pressure depends linearly on the value of applied external force. In the considered case of unit force the optimal inflation decreases the bending moment to $11,72 \mathrm{kNm}$ and thus it increases load capacity of the structure by $58,4 \%$, Figure 23 (bottom).

Further improvement can be achieved by applying internal pressure exclusively in selected parts of the structure. In order to avoid an adverse effect of increase of bending moment at the lower beam, the structure is divided horizontally into two separate pressure chambers and only the upper chamber is subjected to inflation (Figure 24). The length of the upper chamber is considered as a design variable. The equilibrium of considered structure with upper chamber of length $d$ and internal pressure $p$ is described by the equation

$$
\mathbf{K}(d) \mathbf{q}=\mathbf{Q}_{\mathbf{F}}+p \mathbf{Q}_{\mathbf{p}}(d) \text {. }
$$

The corresponding optimisation problem is to find the optimal length of the upper chamber and the optimal value of scalar pressure multiplier:

find $d, p$ such that $\max M(x)$ is minimal.

For each assumed length of the upper chamber the optimal adjustment of internal pressure allows equalising bending moments at the upper beam in its centre and in locations where internal chamber is attached. Optimal value of parameter $d$ was found by testing several types of structures with various lengths of the internal chamber. Finally, the largest reduction of the bending moment (to $4,787 \mathrm{kNm}$ ) and the corresponding largest increase in load capacity (286\%) were obtained in the case when length of the internal chamber equals the total length of the frame, Figure 24.

5.3. Minimisation of Impacting Object Deceleration. The two following numerical examples concern the control of mechanical response of inflatable structure subjected to impact loading. In both cases the controlled quantity is the change of pressure in particular chambers of inflatable structure during the impact process. Obtained results can be further used to determine required inflow rate from inflators and outflow rate through discharge valves.

The first example is related to control of the impacting object deceleration. The problem solved is aimed at maintaining constant, possibly low level of deceleration during the entire process of impact. In this case, the analysed model of the frame is fully nonlinear in terms of geometry and material model. Moreover, it involves separate rigid object modelling colliding body as well as definitions of contact conditions between the object and the barrier and between the upper and lower beam of the frame, Figure 25. 


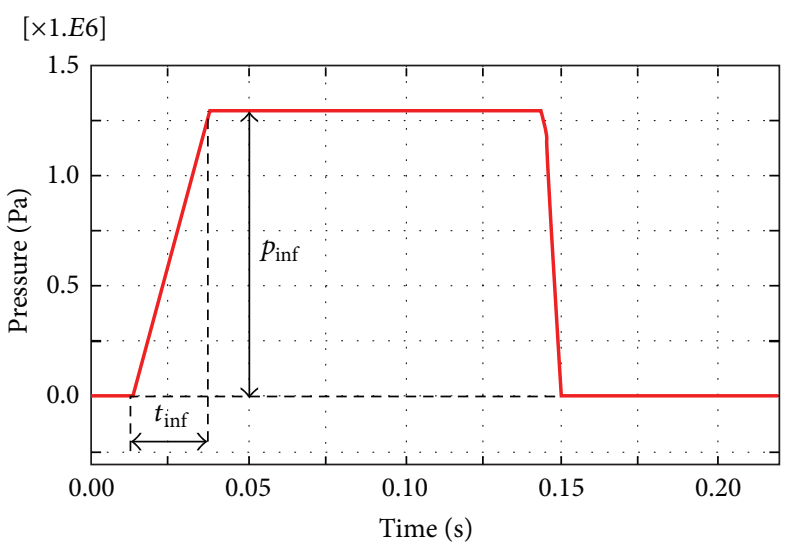

(a)

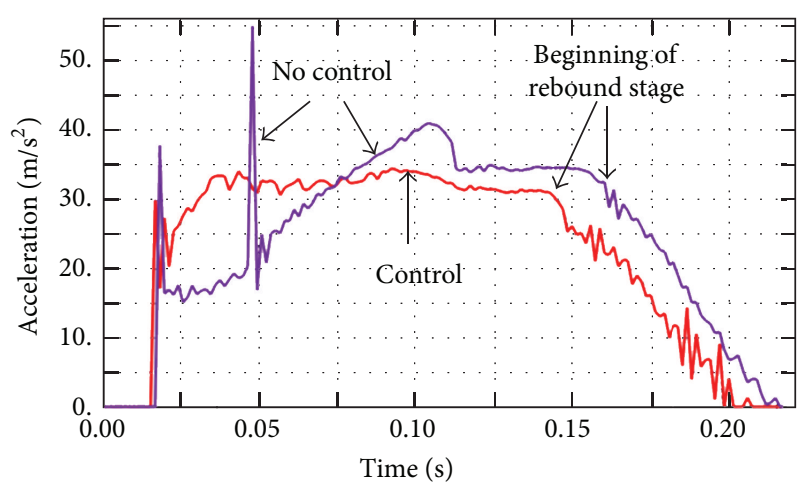

(b)

FIGURE 26: Adjustment of pressure in case of high energy impact: (a) optimal change of pressure in time, (b) comparison of impacting object decelerations in passive and adaptive structure.

In the considered system the time-history of colliding object deceleration depends on whether the collision of both beams of the frame occurs. When impact energy is adequately large, the first peak of deceleration caused by contact of the object and the barrier is followed by the second peak induced by collision of the upper and lower beam and the stage of gradual increase of deceleration being the effect of joint resistance of both beams (Figure 26, "no control"). Therefore, the initial inflation and real-time control of internal pressure will be aimed at mitigation or complete elimination of beam's contact and the following stage of fast deceleration increase.

In order to achieve precise control of the impact process two different control methods were proposed:

(i) the "local approach" based on decomposition of the original problem into a series of simpler problems related to values of pressure in subsequent time instants,

(ii) the "global approach" based on direct searching of the parameters of functions defining change of internal pressure in terms of time.

In the presented numerical example, the second method is applied to single-chamber inflatable barrier. The continuous function describing change of pressure in time $p(t)$ is substituted by two parameters: $t_{\text {inf }}$ : the period of structure inflation and $p_{\text {inf }}$ : the value of pressure which is maintained constant during the impact process, Figure 26(a). Although such formulation is strongly simplified in comparison to the original one, it was found to be very effective for the control of impacting object deceleration. The precise tuning of both introduced parameters to mass and velocity of the colliding object provides a compromise between an increase of stiffness of the upper beam caused by internal pressure during the first stage of impact and an increase of global stiffness of the structure due to collision of both beams.

As a result of applied strategy, the level of impacting object deceleration remains approximately constant during the major part of the impact process, Figure 26(b). Despite the fact that the way of stopping the impacting body is reduced, the level of impacting object deceleration decreases from $55,02 \mathrm{~m} / \mathrm{s}^{2}$ (or $41,5 \mathrm{~m} / \mathrm{s}^{2}$ excluding peak) to $34,9 \mathrm{~m} / \mathrm{s}^{2}$. Let us note that obtaining constant deceleration for various impact scenarios requires separate adjustment (and different resulting values) of inflation parameters.

5.4. Control of Structure Deformation during Impact. Another purpose of pressure control is to obtain predefined mode of structure deformation and desired final configuration of the system, independently of applied loading. In the particular case of considered inflatable thin-walled frame subjected to impact loading the assumed objective is to achieve maximal compression of selected chambers in final configuration. The proposed approach allows controlling the locations of generation of main plastic hinges being the major mechanism of energy dissipation. For such formulated problem the most spectacular results were obtained for five-chamber barrier, where adjustment of internal pressure in selected parts of the structure had enabled precise control of the deformation process.

The corresponding numerical example concerns fivechamber frame exposed central and lateral impact of a rigid object (Figure 27). In particular, in case of strong central impact the noninflated structure is subjected to a large deformation associated with total crushing of the central chamber. However, inflation of the central chamber with initial pressure properly adjusted to impact energy allows avoiding its large compression, distributing deformation into two sides of the frame, and obtaining final configuration involving two main plastic hinges.

In turn, impact applied to left peripheral chamber of noninflated frame causes response involving total crushing of the impacted chamber and insignificant deformation of the remaining part of the frame, Figure 27(a). Nevertheless, accurately adjusted inflation of the left part of the frame causes that the structure becomes sensitive to the value 


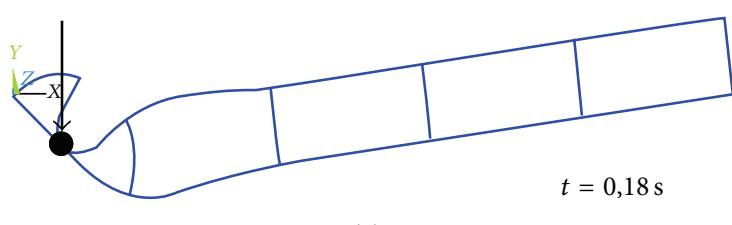

(a)

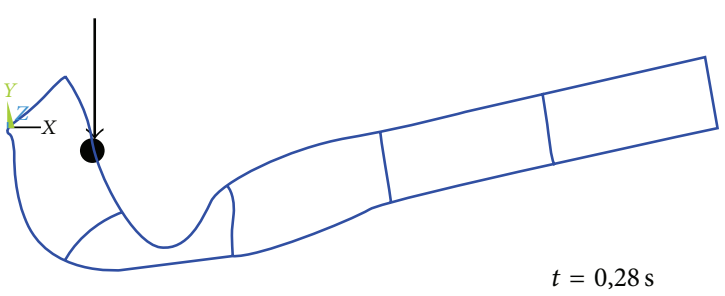

(b)

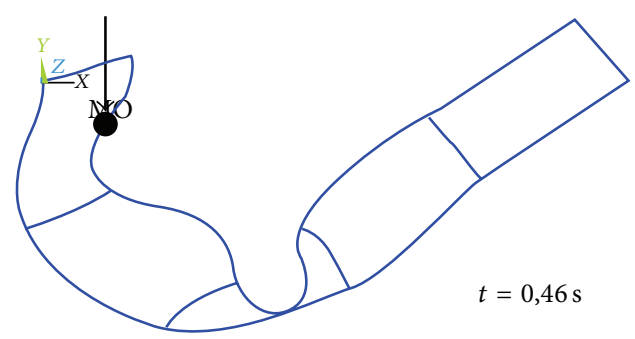

(c)

FIGURE 27: Various deformations of the frame obtained for the case of lateral impact: (a) no inflation, ((b) and (c)) inflation causing change of final deformation shape.

of applied internal pressure and shape of deformation is substantially changed. The precise adjustment of the initial value of pressure and speed of pressure release in the left part of the frame allows controlling the mode of deformation and obtaining either crushing of the second chamber of the frame (Figure 27(b)) or shift of maximal deformation to its central part (Figure 27(c)). Qualitative change of deformation mode is associated with increase of the maximal deflection of the frame and elongation of the deformation period.

\section{Conclusions}

Basic mechanical properties of inflatable thin-walled crashworthy structures filled with compressed gas were analysed and the possibilities of improving their dynamic response to lateral loading were thoroughly investigated. The performed experiment with aluminium beverage can had proved the beneficial influence of initial inflation on the process of buckling caused by transverse loading. Moreover, the corresponding numerical simulations had revealed the influence of internal pressure on initial stress distribution, the value of the buckling force, the buckling shape, and the possibility of can bursting. The following numerical examples, which had concerned various types of inflatable thin-walled structures subjected to impact loading (hull compartments, door of the car, and traffic barrier), had shown several important favourable effects related to application of compressed gas. As it was demonstrated, internal pressure allows preventing total penetration by impacting object, increasing global stiffness of the structure, and, finally, influencing zones of local stress concentration. Eventually, the concept of adaptive inflatable thin-walled barrier was utilised to prove that control of internal pressure can provide adaptation to actual impact loading resulting in favourable stress distribution, constant deceleration of impacting object, and desired final deformation of the structure.

The presented examples indicate that a wide range of mechanical properties of thin-walled structures as well as their durability to lateral impact loading can be significantly enhanced by inflation with compressed gas. The paper proves that inflation of thin-walled structures allows obtaining desired change of their dynamic response and thus it has large potential of application in structural crashworthiness.

\section{Conflict of Interests}

The authors declare that there is no conflict of interests regarding the publication of this paper.

\section{Acknowledgments}

This work was partially financed from the funds of the National Science Centre allocated on the basis of Decisions nos. DEC-2012/05/B/ST8/02971 and DEC-2014/15/B/ST8/ 04363. Mr. Rafał Chmielewski is gratefully acknowledged for conducting the experiment with aluminium beverage can.

\section{References}

[1] J. M. Alexander, "An approximate analysis of the collapse of thin cylindrical shells under axial loading," Quarterly Journal of Mechanics and Applied Mathematics, vol. 13, no. 1, pp. 10-15, 1960.

[2] N. Jones, Structural Impact, Cambridge University Press, 1989.

[3] N. Jones and T. Wierzbicki, Eds., Structural Crashworthiness, Butterworths, London, UK, 1983.

[4] N. Jones and T. Wierzbicki, Eds., Structural Failure, John Wiley \& Sons, New York, NY, USA, 1989. 
[5] N. Jones and T. Wierzbicki, Eds., Structural Crashworthiness and Failure, Elsevier Applied Science, 1993.

[6] J. A. C. Ambrosio, Ed., Crashworthiness of Transportation System: Structural Impact and Occupant Protection, Kluwer Academic Publishers, 1997.

[7] K. J. Bathe, Finite Element Procedures in Engineering Analysis, Prentice Hall, 1981.

[8] O. C. Zienkiewicz, The Finite Element Method, McGraw-Hill, New York, NY, USA, 1997.

[9] LS-DYNA software, http://www.lstc.com/.

[10] J. N. Reddy, Introduction to Nonlinear Finite Element Methods, Oxford Scholarship Online, Oxford, UK, 2004.

[11] T. Belytschko, W. K. Liu, and B. Moran, Nonlinear Finite Elements for Continua and Structures, John Wiley \& Sons, 2000.

[12] K. Wiśniewski and P. Kołakowski, "The effect of selected parameters on ship collision results by dynamic FE simulations," Finite Elements in Analysis and Design, vol. 39, no. 10, pp. 985$1006,2003$.

[13] W. Abramowicz, "Macro element method on crashworthiness of vehicles," in Crashworthiness: Energy Management and Occupant Protection, J. Ambrosio, Ed., Springer, New York, NY, USA, 2001.

[14] H.-S. Kim and T. Wierzbicki, "Effect of the cross-sectional shape on crash behaviour of a three dimensional space frame," International Journal of Vehicle Design, vol. 25, no. 4, pp. 295316, 2001.

[15] H.-S. Kim and T. Wierzbicki, "Effect of the cross-sectional shape of hat-type cross-sections on crash resistance of an 'S'-frame," Thin-Walled Structures, vol. 39, no. 7, pp. 535-554, 2001.

[16] J. Han and K. Yamazaki, "Crashworthiness optimization of Sshape square tubes," International Journal of Vehicle Design, vol. 31, no. 1, pp. 72-85, 2002.

[17] A. A. A. Alghamdi, "Collapsible impact energy absorbers: an overview," Thin-Walled Structures, vol. 39, no. 2, pp. 189-213, 2001.

[18] D. W. Lee, An innovative inflatable morphing body structure for crashworthiness of military and commercial vehicles [Ph.D. thesis], University of Michigan, 2009.

[19] X. Zhang, Conceptual study of adaptive energy absorbers [Ph.D. thesis], The Hong Kong University of Science and Technology, 2009.

[20] M. Ostrowski, P. Griskevicius, and J. Holnicki-Szulc, "Adaptive crashworthiness of front-end structure of motor vehicles," in Proceedings of the SAE World Congress, Detroit, Mich, USA, 2007.

[21] K. Greń, "Dissipation of energy in absorber filled with compressed air," Publications of Institute of Vehicles 4/47, Warsaw University of Technology, Warsaw, Poland, 2002.

[22] B. Pipkorn and Y. Håland, "Proposed variable stiffness of vehicle longitudinal frontal members," International Journal of Crashworthiness, vol. 10, no. 6, pp. 603-608, 2005.

[23] J. Holnicki-Szulc and R. Chmielewski, Polish Patent, P-357761.

[24] C. Graczykowski, R. Chmielewski, and J. Holnicki-Szulc, "Controlled impact absorption in adaptive pressurized structures," in Proceedings of the 4th European Congress on Computational Methods in Applied Sciences and Engineering, Jyväskylä, Finland, July 2004.

[25] Z. Mazurkiewicz, Thin Elastic Shells. Linear Theory, OW PW, Warszawa, Poland, 2004 (Polish).

[26] F. Flugge, Stresses in Shells, Springer, Berlin, Germany, 1973.
[27] M. Amabili and R. Garziera, "Vibrations of circular cylindrical shells with nonuniform constraints, elastic bed and added mass. Part II: shells containing or immersed in axial flow," Journal of Fluids and Structures, vol. 16, no. 1, pp. 31-51, 2002.

[28] M. Amabili and R. Garziera, "Vibrations of circular cylindrical shells with nonuniform constraints, elastic bed and added mass. Part I. Empty and fluid-filled shells," Journal of Fluids and Structures, vol. 14, no. 5, pp. 669-690, 2000.

[29] C. Graczykowski, Inflatable structures for adaptive impact absorption [Ph.D. thesis], IPPT PAN, 2011.

[30] C. Graczykowski, "Mathematical models and numerical methods for the simulation of adaptive inflatable structures for impact absorption," Computers \& Structures, 2015.

[31] V. U. Minorsky, "An analysis of ship collisions with reference to protection of nuclear power ships," Journal of Ship Research, vol. 3, no. 2, 1959.

[32] H. Lenselink and K. G. Thung, "Numerical simulations of the Dutch-Japanese full scale ship collisions tests," The MacNealSchwendler Company Report, MacNeal-Schwendler Company, Gouda, The Netherlands, 1992.

[33] S. Haris and J. Amdahl, "Analysis of ship-ship collision damage accounting for bow and side deformation interaction," Marine Structures, vol. 32, pp. 18-48, 2013.

[34] S. Rastegar, "Adaptive security and protective barriers and traffic security protective bumps," US Patent, 2008/0056818.

[35] R. Gupta and A. D. Kelkar, "Nonlinear crash dynamics sumualtion of novel airbag based next generation energy absorbing barrier," in Proceedings of the 9th International LS-Dyna Users Conference, Michigan, Mich, USA, 2006.

[36] J. Holnicki-Szulc, C. Graczykowski, G. Mikułowski, A. Mróz, and P. Pawłowski, "Smart technologies for adaptive impact absorption," Solid State Phenomena, vol. 154, pp. 187-194, 2009.

[37] G. Mikułowski, P. Pawłowski, C. Graczykowski, R. Wiszowaty, and J. Holnicki-Szulc, "On a pneumatic adaptive landing gear system for a small aerial vehicle," in Proceedings of the 4th Eccomas Thematic Conference on Smart Structures and Materials, Porto, Portugal, 2009.

[38] C. Graczykowski and J. Holnicki-Szulc, "Protecting offshore wind turbines against ship impacts by means of adaptive inflatable structures," Shock and Vibration, vol. 16, no. 4, pp. 335353, 2009.

[39] K. Sekuła, C. Graczykowski, and J. Holnicki-Szulc, "On-line impact load identification," Shock and Vibration, vol. 20, no. 1, pp. 123-141, 2013. 


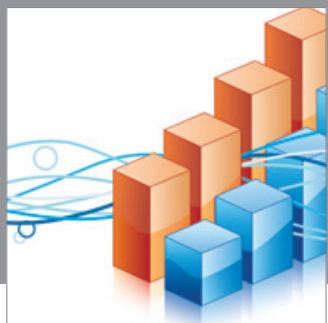

Advances in

Operations Research

mansans

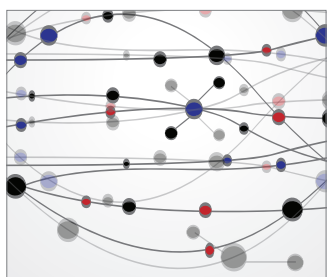

The Scientific World Journal
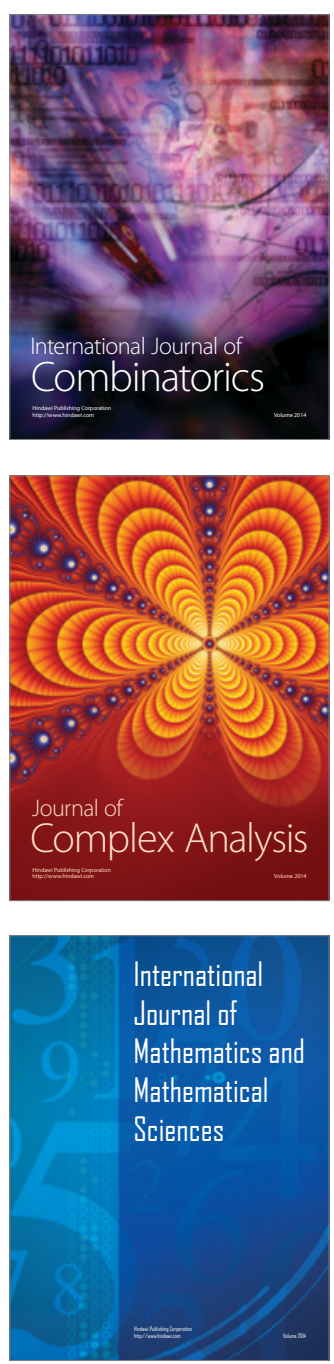
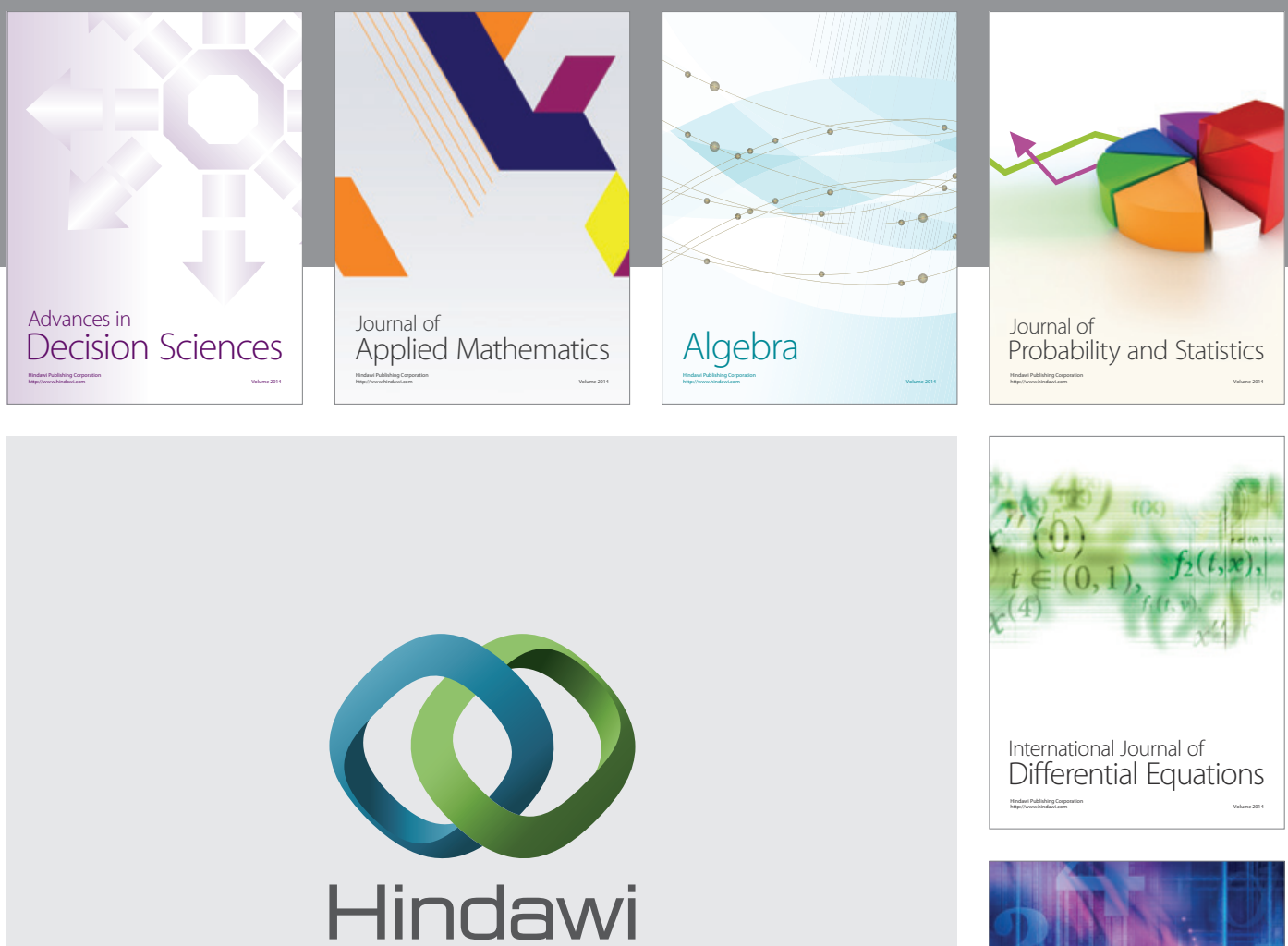

Submit your manuscripts at http://www.hindawi.com
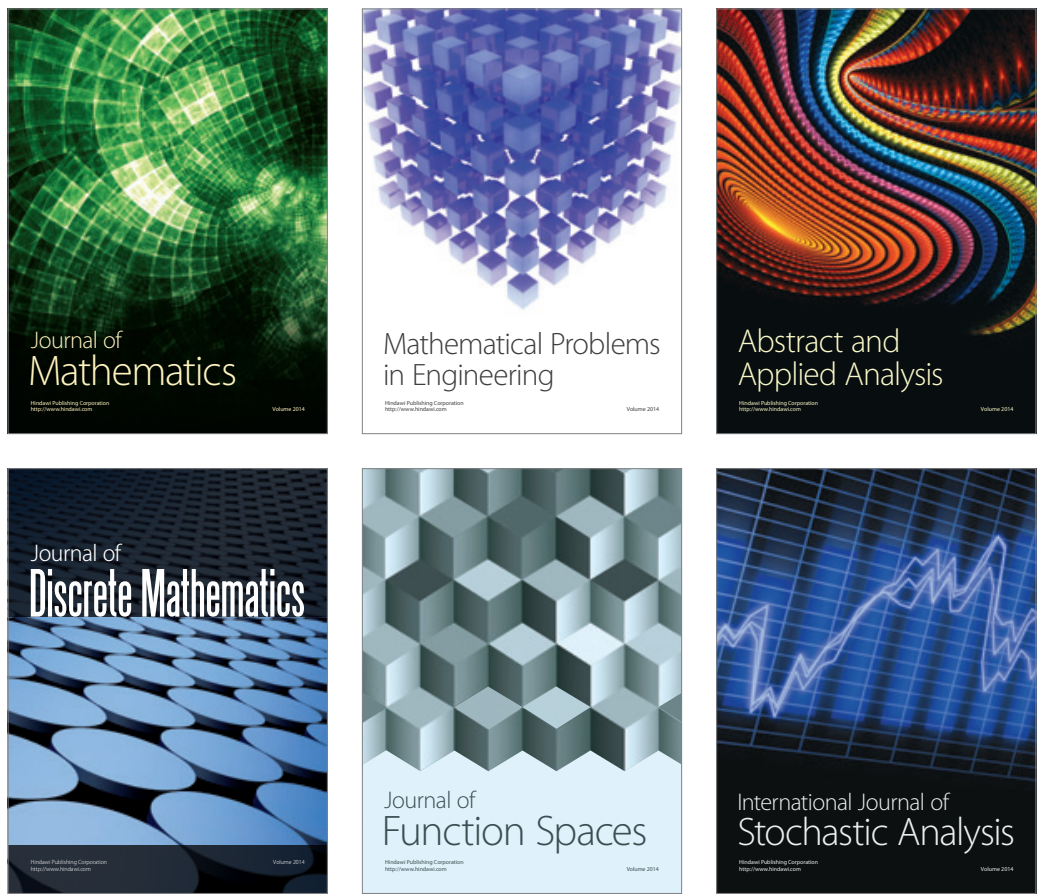

Journal of

Function Spaces

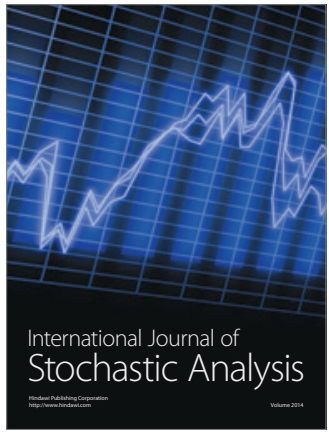

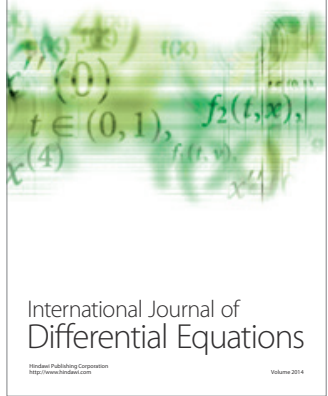
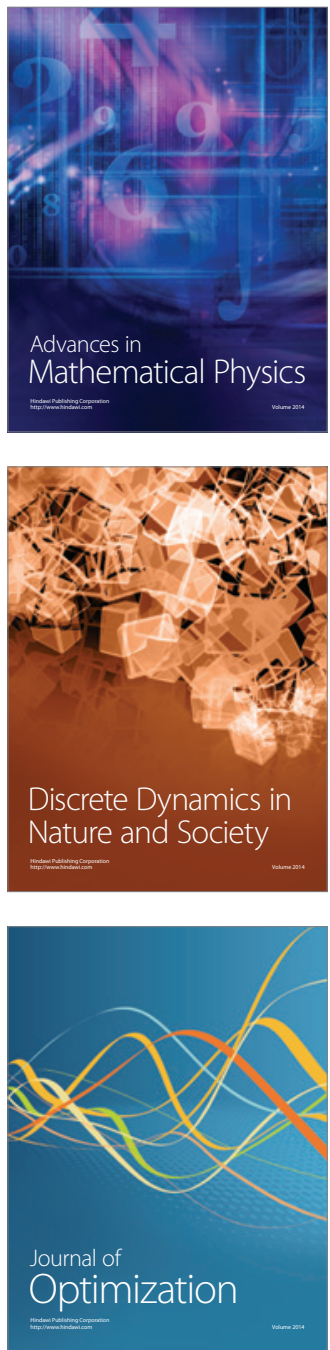